\title{
Evaluating Cloud Service Elasticity Behavior
}

\author{
Georgiana Copil*, Hong-Linh Truong, Daniel Moldovan \\ and Schahram Dustdar \\ Distributed Systems Group, Vienna University of Technology \\ Argentinierstrasse 8, A-1040 Vienna, Austria \\ *e.copil@dsg.tuwein.ac.at
}

Demetris Trihinas, George Pallis and Marios D. Dikaiakos

Department of Computer Science, University of Cyprus

P. O. Box 20537, 1678 Nicosia, Cyprus

Received 14 February 2015

Accepted 21 July 2015

Published 7 September 2015

\begin{abstract}
To optimize the cost and performance of complex cloud services under dynamic requirements, workflows and diverse cloud offerings, we rely on different elasticity control processes. An elasticity control process, when being enforced, produces effects in different parts of the cloud service. These effects normally evolve in time and depend on workload characteristics, and on the actions within the elasticity control process enforced. Therefore, understanding the effects on the behavior of the cloud service is of utter importance for runtime decision-making process, when controlling cloud service elasticity.

In this paper, we present a novel methodology and a framework for estimating and evaluating cloud service elasticity behaviors. To estimate the elasticity behavior, we collect information concerning service structure, deployment, service runtime, control processes, and cloud infrastructure. Based on this information, we utilize clustering techniques to identify cloud service elasticity behavior, in time, and for different parts of the service. Knowledge about such behavior is utilized within a cloud service elasticity controller to substantially improve the selection and execution of elasticity control processes. These elasticity behavior estimations are successfully being used by our elasticity controller, in order to improve runtime decision quality. We evaluate our framework with three real-world cloud services in different application domains. Experiments show that we are able to estimate the behavior in $89.5 \%$ of the cases. Moreover, we have observed improvements in our elasticity controller, which takes better control decisions, and does not exhibit control oscillations.
\end{abstract}

Keywords: Elasticity; elasticity behavior; clustering.

\section{Introduction}

With the wide adoption of cloud computing across multiple business domains, stakeholders seek to improve the efficiency of their complex cloud services, while also if possible to reduce costs, by acquiring on-demand virtualized infrastructure and, at the same time, benefiting from a pay-as-you-go price model offered by cloud

* Corresponding author. 


\section{G. Copil et al.}

providers. The key technique to achieve these goals is elasticity ${ }^{1,2}$ — the ability of cloud services to acquire and release resources on-demand, in response to runtime fluctuating workloads. From the customer perspective, resource elasticity can minimize task execution time, without exceeding a given budget. From the cloud provider perspective, elasticity provisioning contributes to maximizing their financial gain while keeping their customers satisfied and reducing administrative costs. However, automatic elasticity provisioning is not a trivial task.

To date, the user utilizes elasticity controllers, offered as a service, by either cloud providers (e.g. Amazon Auto Scalinga) or third-party vendors (e.g. Rightscale $^{\mathrm{b}}$ ), to scale his/her distributed cloud services. A common approach, employed by many elasticity controllers, ${ }^{3,4}$ is to monitor the cloud service and (de-)provision virtual instances for the service when metric thresholds are violated. This approach may be sufficient for simple cloud services, but for large-scale distributed cloud services with complex inter-dependencies among components, we need a deeper understanding of their elasticity behavior in order to select and enforce suitable elasticity control processes. For this reason, existing work $^{4,5}$ has identified a number of elasticity control processes to improve the performance and quality of cloud services, while additionally attempting to minimize cost. However, a crucial question still remains unanswered: Which elasticity control processes are the most appropriate for a cloud service in a particular situation at runtime? Moreover, can both cloud customers and providers benefit from insightful information such as how the addition of a new instance to a cloud service will affect the throughput of the overall deployment and individually each part of the cloud service? Thus, cloud service elasticity behavior knowledge under various controls and workloads is of paramount importance to elasticity controllers for improving their runtime decision making.

To this end, a wide range of approaches relying on service profiling or learning from historic information were proposed. ${ }^{6,7}$ However, these approaches limit their decisions to evaluating only low-level VM metrics (e.g. CPU and memory usage) and do not support elasticity decisions considering complex cloud service behavior at multiple levels (e.g. a specific part of the service or the entire service). Additionally, current approaches only evaluate resource utilization, without considering elasticity as a multi-dimensional property composed of cost, quality, and resource elasticity. Finally, existing approaches do not consider the outcome of a control process on the overall service behavior, where often enforcing a control process on the wrong part of the cloud service can lead to side effects, such as increasing the cost or decreasing performance of the overall service.

In this paper, we focus on addressing the previous limitations by introducing a methodology for estimating cloud service elasticity behavior, and a corresponding framework named evAluating clouD serVIce elaSticity bEhavior (ADVISE). Our

${ }^{\text {a http://aws.amazon.com/autoscaling/. }}$

${ }^{\mathrm{b}}$ http://www.rightscale.com/. 
behavior estimation technique introduces a clustering-based process which considers heterogeneous information for computing expected elasticity behavior, in time, for various service parts. To estimate cloud service elasticity behavior, ADVISE utilizes different types of information, such as service structure, deployment strategies, and underlying infrastructure dynamics, when applying different workload and elasticity control processes. ADVISE analyzes historical cloud service behavior, at various levels of abstraction, and produces estimations for elasticity control processes evaluated by the elasticity controller, in time, and for all cloud service parts, not only for the one targeted by the elasticity control process.

For validating our techniques, we integrate ADVISE in $\mathrm{rSYBL}^{8}$ elasticity controller. rSYBL is based on SYBL elasticity requirements specification language, ${ }^{9}$ which allows service providers to describe invariants and expected service behavior. rSYBL interprets requirements specified in SYBL, and based on these requirements it provides multi-grain elasticity control for complex cloud services. To evaluate ADVISE effectiveness, experiments were conducted on two cloud platforms with a testbed comprised of three cloud services originating from different service domains. Results show that ADVISE is able to determine the expected elasticity behavior, in time, with a low error rate (i.e. average standard deviation 0.46 over all considered elasticity control processes). Therefore, ADVISE can be integrated by cloud providers alongside their elasticity controllers to improve the decision quality, or used by service providers to evaluate and understand how various elasticity control processes impact their offered services. ADVISE and all the other tools used in this paper (i.e. rSYBL, JCatascopia, ${ }^{10}$ and MELA ${ }^{11}$ ) are available as open source tools, thus enabling various stakeholders to apply them, or if needed to extend them, for obtaining elasticity in their respective domains.

This paper substantially extends and details our previous work, ${ }^{12}$ as follows: (i) We detail the methodology used to provide elasticity behavior estimation; (ii) we integrate ADVISE with the rSYBL elasticity controller, and detail the new architecture; (iii) we design new decision mechanisms for controlling a service based on the elasticity behavior evolution, in time, as opposed to discrete values; (iv) we present further experiments on ADVISE elasticity behavior estimation; and finally, (v) we present new experiments showing ADVISE-driven control decisions.

The rest of this paper is structured as follows: In Sec. 2, we model relevant information regarding cloud services. In Sec. 3, we present the elasticity behavior evaluation process. In Sec. 4, we present how ADVISE is integrated into rSYBL elasticity controller. In Sec. 5, we evaluate ADVISE framework effectiveness. In Sec. 6, we discuss related work and Sec. 7 concludes this paper.

\section{Cloud Service Structural and Runtime Information}

\subsection{Cloud service information}

Following existing common service descriptions, ${ }^{13,14}$ in our study we refer to cloud applications, platforms, and systems as cloud services. A cloud service can be 


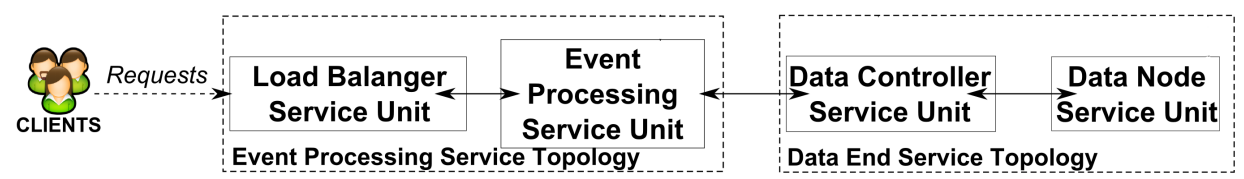

Fig. 1. An M2M DaaS topology structure.

decomposed into different parts such as individual service units and topologies of units; in this paper we use the term Service Parts (SP) to refer them. For understanding the behavior of cloud services, we must gather multiple types of information, including application-specific behavior for different service parts and the various virtual resources used, and their characteristics. Taking for instance a M2M DaaS cloud service, as depicted in Fig. 1 and used in our experimental evaluation (see Sec. 5), the M2M DaaS is composed of multiple service units: An event processing unit, a load balancer unit, a data node unit and a data controller unit. The first two units are grouped into a service topology, which processes incoming events (i.e. EventProcessingServiceTopology), while events are stored into the DataEndService Topology, composed of the latter two units.

To represent such above-mentioned complex cloud services, we extend the conceptual cloud service representation model, as proposed in Ref. 8, with a rich set of information types for determining cloud elasticity behavior. Figure 2 depicts the extensions made (white background) to include elasticity control processes, service part behaviors and service parts. Overall, our information model contains: (i) Structural information, describing the architectural structure of the

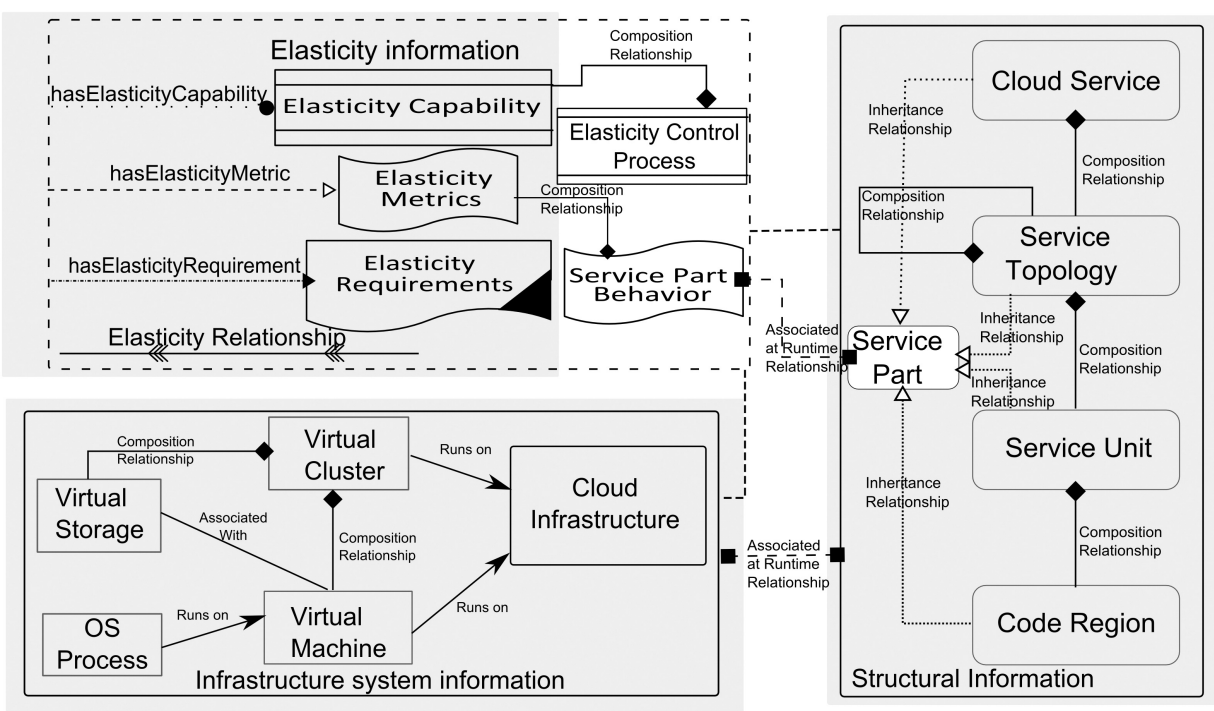

Fig. 2. Cloud service information for estimating elasticity behavior. 
cloud service; (ii) infrastructure system information, describing runtime information regarding resources allocated for the cloud service by the underlying cloud platform; and (iii) elasticity information, capturing elasticity metrics, requirements, and capabilities.

Elasticity information is composed of elasticity metrics (e.g. average response time, cost, active connections), elasticity requirements (e.g. minimize response time when cost is small enough), and elasticity capabilities (e.g. add new resources), each of them being associated to different SPs or infrastructure resources. Elasticity capabilities are grouped together as elasticity control processes (ECPs), as described in the next subsection, and inflict specific elasticity behaviors upon enforcement on different SPs, which we model as Service Part Behaviors. We model SP behaviors, since controllers must determine the effect of enforcing an ECP at different levels. For instance, in the service previously described, before allocating a new DataNodeServiceUnit instance, the effect, in time, at the DataEndServiceTopology (e.g. latency evolution for the entire cluster), and at the entire cloud service level (e.g. number of violated requirements while enforcing the ECP) should be determined.

Conceptually, a Service Part Behavior, denoted as Behavior $\mathrm{SP}_{i}$, in a defined period of time [start, end], contains all the metrics, $M_{a}^{\mathrm{SP}}{ }_{i}$, being monitored for $\mathrm{SP}_{i}$.

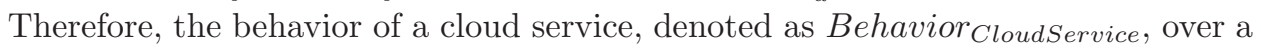
period of time is defined as the set of all cloud service SP behaviors:

$$
M_{a}^{\mathrm{SP}_{i}}[\text { start }, \text { end }]=\left\{M_{a}\left(t_{j}\right) \mid \mathrm{SP}_{i} \in \text { ServiceParts }, j=\overline{\text { start }, \text { end }}\right\},
$$

$$
\begin{aligned}
& \text { Behavior }_{\mathrm{SP}_{i}}[\text { start }, \text { end }]=\left\{M_{a}^{\mathrm{SP}_{i}}[\text { start }, \text { end }] \mid M^{a} \in \operatorname{Metrics}\left(\mathrm{SP}_{i}\right)\right\}, \\
& \text { Behavior }_{\text {CloudService }}[\text { start }, \text { end }]=\left\{\text { Behavior }_{\mathrm{SP}_{i}}[\text { start }, \text { end }] \mid\right. \\
&\left.\mathrm{SP}_{i} \in \operatorname{ServiceParts}(\text { CloudService })\right\} .
\end{aligned}
$$

The above information is captured and managed at runtime through an elasticity dependency graph, $\mathrm{EDG}=\{(V, E) \mid V \in \mathrm{SP} \cup$ InfrastructureInfo, $E \in$ Relationships\}, which has as nodes instances of concepts from the model in Fig. 2 (e.g. Virtual Machine, Elasticity Metric), and relationships (e.g. Elasticity Relationship, Inheritance, Composition) as edges. The elasticity dependency graph is continuously updated with: (i) pre-deployment information, such as service topology descriptions (e.g. TOSCA ${ }^{13}$ ) or profiling information and (ii) runtime information, such as metric values from monitoring tools or allocated resources from provider APIs.

\subsection{Elasticity capabilities and control processes}

Elasticity capabilities (ECs) are the set of actions associated with a cloud service, whose invocation affect the cloud service behavior. Such capabilities can be exposed by: (i) Different SPs (e.g. change refresh rate for a SP); (ii) cloud providers (e.g. create new virtual resources); and (iii) resources which are supplied by cloud 


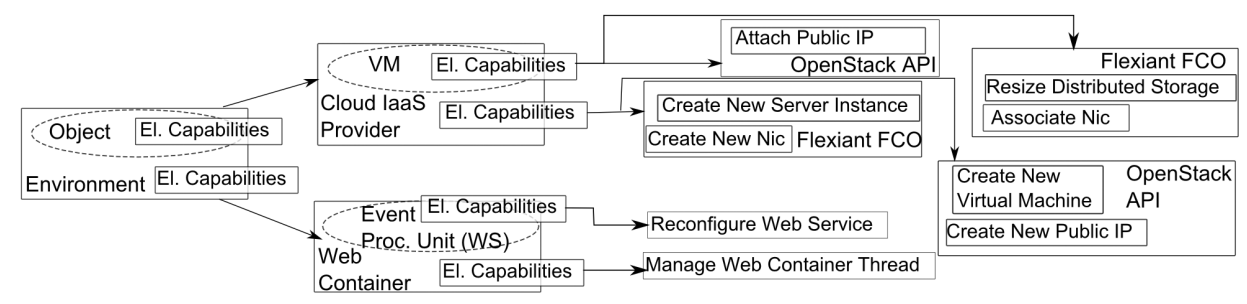

Fig. 3. Elasticity capabilities exposed by different elastic objects.

providers (e.g. change virtual resource characteristics). An EC can be considered as the abstract representation of API calls, which differ amongst providers and cloud services. Figure 3 depicts the different subsets of ECs provided for the Event Processing Service Unit (i.e. a web service hosted in a web container) when deployed on two different cloud platforms (e.g. Flexiant ${ }^{c}$ and Openstack ${ }^{\mathrm{d}}$ ), as well as the ECs exposed by the cloud service and its containers (e.g. Apache Tomcat). In each of the two cloud platforms, the cloud service must run on a specific container, and all these capabilities, when enforced by an elasticity controller, will affect various cloud service parts (e.g. in the M2M DaaS, elasticity capabilities of Event Processing Service Unit might affect the performance of the Data End Service Topology).

Elasticity Control Processes (ECP) are processes composed of elasticity capabilities, which can be abstracted into higher level capabilities having predictable effects on the cloud service. ECPs can be in their simplest forms, sequential elasticity capabilities, while the more complex ECPs are similar to business processes (e.g. enforcement plans from TOSCA described in BPMN). We model these ECPs as graphs, $\mathrm{ECP}=(V=\{\mathrm{EC}\}, E=\{\mathrm{CF}, \mathrm{DF}\})$, where the vertices are elasticity capabilities, while edges are flow dependencies among elasticity capabilities. There are two types of flow dependencies: (i) Control flow (CF) dependencies, which direct the execution of the process considering the initial state and (ii) data flow (DF) dependencies, which carry data to be used by the next EC.

An ECP causes a change in the elasticity dependency graph and in the virtual infrastructures (e.g. a change in the properties of a single VM or tier). For example, in the case of a distributed database backend which is composed of multiple nodes, a scale out ECP, with certain parameters, can be applied for both a Cassandra and an HBase database, with the following ECs: (i) add a new node; (ii) configure node properties; and (iii) subscribe node to the cluster.

\subsection{Cloud service elasticity}

To estimate the effects of ECPs on SPs, we rely on the elasticity dependency graph which captures all the variables that contribute to cloud service elasticity behavior

${ }^{\mathrm{c}}$ http://www.flexiant.com/.

d https://www.openstack.org/. 


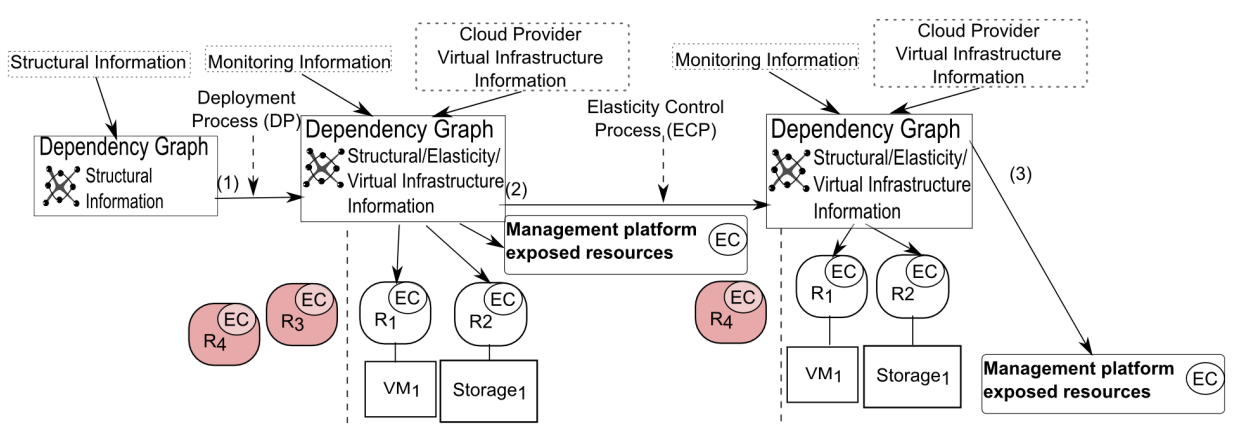

Fig. 4. Elastic cloud service evolution.

evolution. Figure 4 depicts on the left-hand side the cloud service at pre-deployment time, where automatic elasticity controllers know about it only from structural information provided by different sources (e.g. TOSCA description). After enforcing a Deployment Process (e.g. create VM, create network interface and connect to VPN), the elasticity dependency graph will be updated with infrastructure-related information obtained from the cloud provider, and elasticity information, obtained from monitoring services showing metric evolution for different SPs.

Infrastructure resources, as mentioned previously, have associated elasticity capabilities (EC in Fig. 4), that describe the change(s) to be enforced and the mechanisms for triggering them (e.g. API call assigned to the EC). In addition, a cloud platform exposes ECs in order to create new resources or instantiate new services (e.g. increase memory is an EC exposed by a VM, while create new VM is an EC exposed by the cloud platform). In this context, for being able to discover the effects that an ECP produces in time, for each SP, taking into account correlations between metrics, we use the elasticity dependency graph. We analyze this information to determine the effect of an ECP for all SPs, regardless on whether the ECP is application specific, or it does not have any apparent direct link to other SPs.

\section{Evaluating Cloud Service Elasticity Behavior}

Existing behavior learning solutions ${ }^{6,7}$ learn discrete metric models, without correlating metrics with the multiple variables affecting cloud service behavior. In contrast to these solutions, we provide behavior learning based on different SPs and their relation to multiple ECPs, which may or may not be directly linked, estimating the effect of an ECP, in time, considering correlations among several metrics and SPs. Figure 5 depicts the SP behavior Learning Process which is continuously executed, refining the previously gathered knowledge base.

\subsection{Obtaining necessary information}

For evaluating cloud service elasticity behavior, we populate the dependency graph, described in Sec. 2, with all the necessary information (i.e. service parts, elasticity 


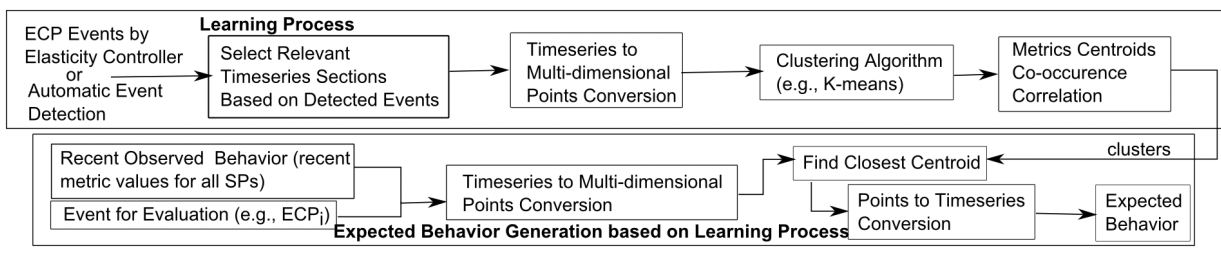

Fig. 5. Modeling cloud service behavior process.

relationships, infrastructure system information). First, we acquire pre-deployment information to understand the cloud service and its execution environment, such as: (i) Structural information, regarding the topology of the cloud service and (ii) cloud infrastructure information. The first, described in Sec. 2, is generally known by the service provider, and contains the SPs of the service and relationships which appear among them. The latter describes virtual resources available in the current cloud infrastructure and their capabilities (e.g. a virtual machine of type $\mathrm{x}$ exposes the capability of memory ballooning). Afterwards, runtime information regarding the service behavior, is collected via monitoring tools (e.g. using MELA ${ }^{11}$ ), and associated with structural service units or topologies.

Monitoring data is either collected at runtime while a controller is enforcing different ECPs, or through a profiling step, where both rational and incorrect ECPs are enforced. In both cases, issues may arise, such as application failure or virtual resource failure, leading to incomplete monitoring data. Therefore, we acknowledge two issue types: (i) recurring issues, which characterize a control step (e.g. a capability) and must be considered and (ii) random issues which do not affect the behavior of a service. The first issue type must be reflected in the estimations, since they characterize the behavior of the service (e.g. while enforcing $\mathrm{ECP}_{i} \mathrm{SP}_{j}$ cannot be monitored for $X \mathrm{~s}$ ). However, the second issue type can be ignored. For this, the following clustering methodology considers both, characterizing recurring behaviors (e.g. missing measurements each time a type $e_{x}$ reconfiguration is enforced) and filtering outliers (e.g. random issues).

\subsection{Learning process}

Figure 6 depicts the overall elasticity behavior clustering process via selected metrics observations, with the three main steps: (a) input data processing, (b) clustering process, and (c) behavioral clusters update.

\subsubsection{Processing input data}

The learning process receives as input each metric's evolution, in time, $M_{a}^{\mathrm{SP}_{i}}[$ start, current] (see Eq. (3)) starting from the beginning of a service's lifecycle. To evaluate the expected metric evolution in response to enforcing a specific ECP, we select for each monitored metric, of each service part, a Relevant Timeseries Section (RTS) (see Fig. 7), in order to compare it with previously encountered 


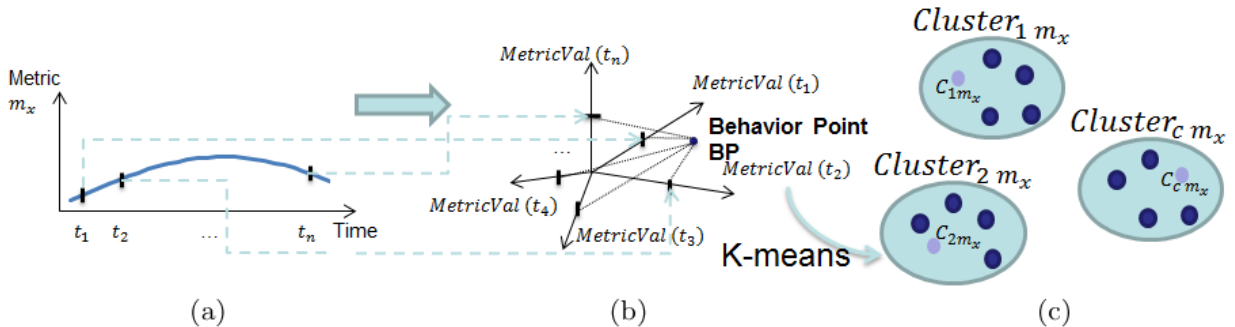

Fig. 6. Clustering process.

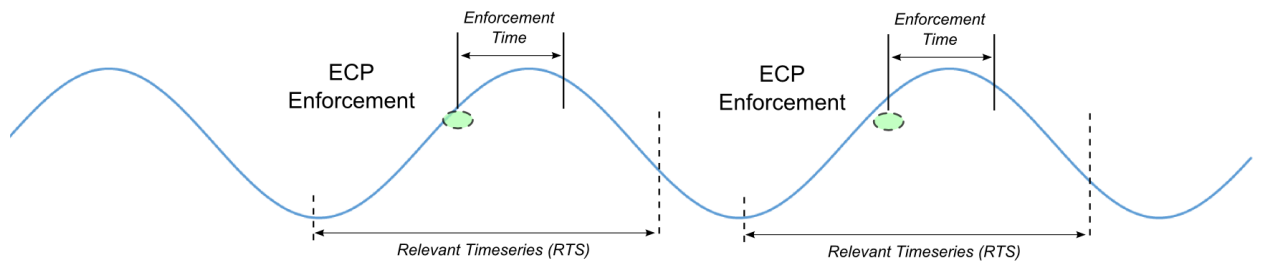

Fig. 7. Relevant timeseries selection.

$M_{a}^{\mathrm{SP}_{i}}[$ start, current]. The RTS size strongly depends on the average time required to enforce an ECP (see Sec. 5.2.1). Consequently, a metric RTS is a sub-sequence of $M_{a}^{\mathrm{SP}_{i}}$, ranging from an interval before and after ECP enforcement:

$$
\begin{aligned}
& \operatorname{RTS}_{M_{a}}^{\mathrm{SP}_{i}}=M_{a}^{\mathrm{SP}_{i}}\left[x-\frac{\delta+\mathrm{ECP}_{\text {time }}}{2}, x+\frac{\delta+\mathrm{ECP}_{\text {time }}}{2}\right], \\
& {\left[\mathrm{ECP}_{\text {startTime }}, \mathrm{ECP}_{\text {endTime }}\right] \subset\left[x-\frac{\delta+\mathrm{ECP}_{\text {time }}}{2}, x+\frac{\delta+\mathrm{ECP}_{\text {time }}}{2}\right],}
\end{aligned}
$$

where $x$ is the ECP index and $\delta$ is the length of the period we aim to evaluate.

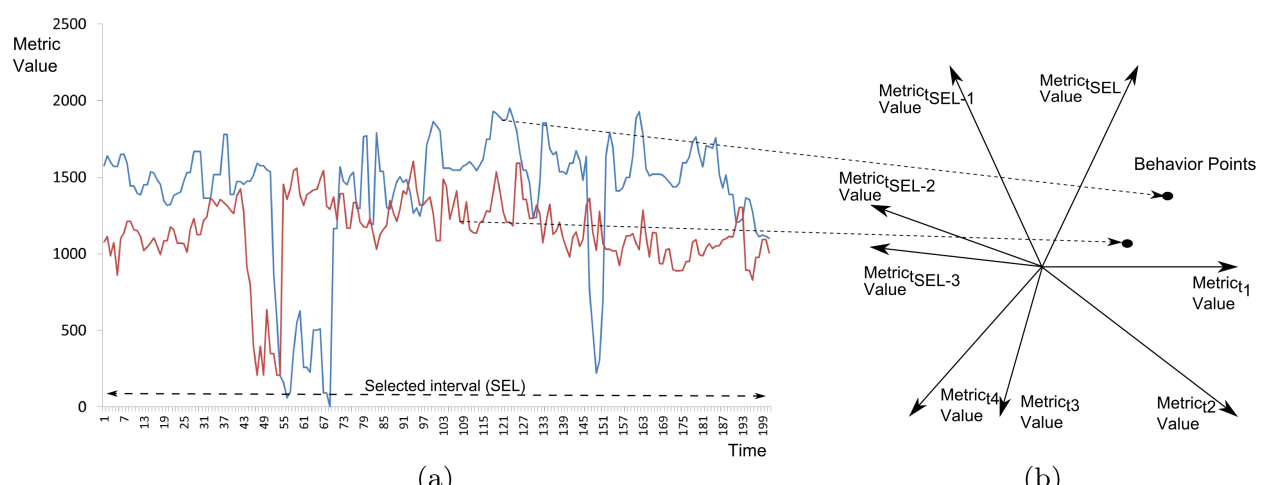

Fig. 8. Relevant timeseries sections to points. 


\section{G. Copil et al.}

As part of the input pre-processing phase, we represent $\delta+\mathrm{ECP}_{\text {time }}$ (Fig. 8(a)) as multi-dimensional points (Fig. 8(b) and Eq. (5)) in the $n$-dimensional Euclidian space, where the value for dimension $t(j)$ is the timestamp $j$ of the current RTS.

$$
\mathrm{BP}_{a}^{\mathrm{SP}_{i}}[j]=\operatorname{RTS}_{M_{a}}^{\mathrm{SP}_{i}}[t(j)], \quad j=0, \ldots, n, \quad \mathrm{BP}: M^{\mathrm{SP}} \mapsto R^{n}, \quad n=\delta+\mathrm{ECP}_{\text {time }}
$$

\subsubsection{Clustering process}

To detect the expected behavior of an ECP enforcement result, we construct behavioral point clusters Cluster $_{\mathrm{SP}_{i}}$, for all SPs and each ECP as defined in Eq. (6). We do not limit our approach to only considering ECPs available for the current $\mathrm{SP}_{i}$ since, as previously mentioned, enforcing an ECP to a specific SP may affect the behavior of another SP or the overall cloud service. Our objective function is to find the multi-dimensional behavior point $C\left(\Theta^{*}\right)$, which minimizes the distance among points belonging to the same cluster Cluster $_{k}$ (Eq. (7)). Since our focus is not to evaluate the quality of different clustering algorithms, we use the $K$-means algorithm, which is inexpensive, following the practice where the number of clusters is $K=\sqrt{N / 2}, N$ being the number of objects. However, as shown in Sec. 5, even with a simple $K$-means algorithm, our approach outputs the expected elasticity behavior with a low estimation error rate.

$$
\begin{aligned}
& \operatorname{dist}\left(\mathrm{BP}_{a}^{x}, \mathrm{BP}_{a}^{y}\right)=\sqrt{\sum_{i}\left(\mathrm{BP}_{a}^{x}[i]-\mathrm{BP}_{a}^{y}[i]\right)^{2}}, \\
& \Theta^{*}=\arg \min \sum_{k=0}^{K} \sum_{i=0}^{N} \Theta_{i, k} \operatorname{dist}\left(\text { Cluster }_{k}, \mathrm{BP}_{i}\right), \\
& \theta_{i, k}= \begin{cases}1 & \mathrm{BP}_{i} \in \text { Cluster }_{k} \\
0 & \mathrm{BP}_{i} \notin \text { Cluster }_{k} .\end{cases}
\end{aligned}
$$

\subsubsection{Behavioral clusters update}

For the update process, we start from the already existing clusters, and we search for new behavior points given by new ECP enforcements. We select relevant timeseries RTS for each SP in response to newly enforced ECPs, whenever new data is available, according to the process presented in Sec. 3.2.1. We represent these as behavior points BP, and add each of them to the closest clusters. The cluster update process then consists of moving BPs among the clusters until convergence, which is a lightweight process compared to running entire clustering algorithm. The overhead of updating the clusters is proportional with the number of selected RTSs and the change in cloud service behavior. Even with ECPs enforced very often, the cluster updating process is still insignificant due to the fact that the RTS dimensions are quite small compared to the overall monitoring data. However, it must 
be noted that repeated ECPs in short intervals reflect a weakness in the controller, which does not manage to stabilize the system as we can see in Sec. 5 .

\subsection{Determining the expected elasticity behavior}

\subsubsection{Elasticity behavior correlation}

After obtaining $\left\|\delta+\mathrm{ECP}_{\text {time }}\right\|$-dimensional point clusters, we construct for each $\mathrm{SP}_{i}$ a co-occurence matrix $\mathrm{CM}_{\mathrm{SP}_{i}}\left[C_{x}^{m_{i}}, C_{y}^{m j}\right]$, where $C_{x}$ is the centroid for Cluster $_{x}$, and the value of $\mathrm{CM}$ is the probability of clusters from metrics $m_{i}$ and $m_{j}$ to appear together (e.g. increase in data reliability is usually correlated with increase in cost). By taking this into consideration, when determining expected behavior points, we have a better estimation which is also based on correlation among metrics (e.g. when in M2M DaaS the EventProcessingServiceUnit throughput is high, a Scale IN ECP will increase response time, while when low, the impact might be negligible). An item in the CM represents a ratio between the number of times the behavior points $C_{x}$ and $C_{y}$ were encountered together and the total number of behavior points. This matrix is continuously updated when behavior points move from one cluster to another, or when new ECPs are enforced, thus, increasing the knowledge base.

\subsubsection{Expected behavior point determination}

In the Expected Behavior Generation based on Learning Process step in Fig. 5, we select the latest metric values for each $\mathrm{SP}_{i}, M_{a}^{\mathrm{SP}_{i}}[$ current $-\delta$, current $]$, and the $\mathrm{ECP}_{\xi}$ which the controller is considering for enforcement, or for which the user would like to know the effects. We find the ExpectedBehavior (see Eq. (8)) which consists of a tuple of cluster centroids from the clusters constructed during the Learning Process that are the closest to the current metrics behavior for the part of the cloud service we are focusing on, and which have appeared together throughout the execution of the cloud service. The result of this step, for each metric of $\mathrm{SP}_{i}$, is a list of expected values from the enforcement of $\mathrm{ECP}_{\xi}$ (e.g. all of the expected metric values for the case the elasticity controller would like to perform a scale out $\mathrm{ECP})$

$$
\begin{aligned}
& \text { ExpectedBehavior }\left[\mathrm{SP}_{i}, \text { Behavior } \operatorname{SP}_{i}[\text { current }-\delta, \text { current }], \mathrm{ECP}_{\xi}\right] \\
& \quad=\left\{C_{i_{a^{1}}}^{M_{a^{1}}}, \ldots, C_{i_{a} m}^{M_{a^{m}}} \mid M_{a^{m}} \in \operatorname{Metrics}\left(\mathrm{SP}_{i}\right)\right\} .
\end{aligned}
$$

\subsection{Parameterizing and qualifying the learning process}

The quality of the expected elasticity behavior estimation depends on several, highly configurable, variables, which are either: (i) The output of a pre-profiling process or (ii) empirically determined, based on good practices or on observing the behavior of the estimation process. Such variables include: (i) Variable $K$, denoting the number 


\section{G. Copil et al.}

of expected clusters; (ii) the variable cutoff, denoting the acceptable clustering convergence error; and (iii) the monitoring information completeness. For $K$, as defined above, we follow the rule of thumb proposed by Mardia et al., ${ }^{15}$ stating that the number of clusters in a set of objects is $\sqrt{N / 2}$, where $N$ is the total number of objects. The offset is empirically determined, considering the quality of the results and the time needed for computing an estimation, as shown in Sec. 5.2.6.

\section{Controlling Elasticity with Elasticity Behavior Estimation}

We have implemented our elasticity estimation techniques in a framework named ADVISE. As described in Sec. 2, ADVISE collects the following heterogeneous types of information, from plugins which we have developed, to populate the elasticity dependency graph: (i) Cloud service structural information, from TOSCA service descriptions; (ii) infrastructure and application performance information, from bothJ Catascopia ${ }^{10}$ and MELA ${ }^{11}$ monitoring systems; and (iii) elasticity information, regarding ECPs from the $\mathrm{rSYBL}^{8}$ elasticity controller.

We extend rSYBL to integrate ADVISE to support both understanding the behavior of the different service parts and enforcing control processes in accordance. Figure 9 depicts the rSYBL framework integrated with ADVISE for estimating elasticity behavior and considering it when generating ECP plans. The rSYBL Elasticity Control Engine, now features two planning mechanisms: (i) profiling-based control, enabled when ADVISE-based estimations are not yet accurate enough (e.g. average standard deviation is low) and (ii) elasticity behavior-based control, which considers runtime elasticity behavior estimations for all service parts in order to evaluate the needed elasticity control process(es). Moreover, ADVISE also exposes statistics on the time required for an ECP to be enforced, thus being able to refine the enforcement cool-off period on the controller side as presented in Sec. 5.2.1.

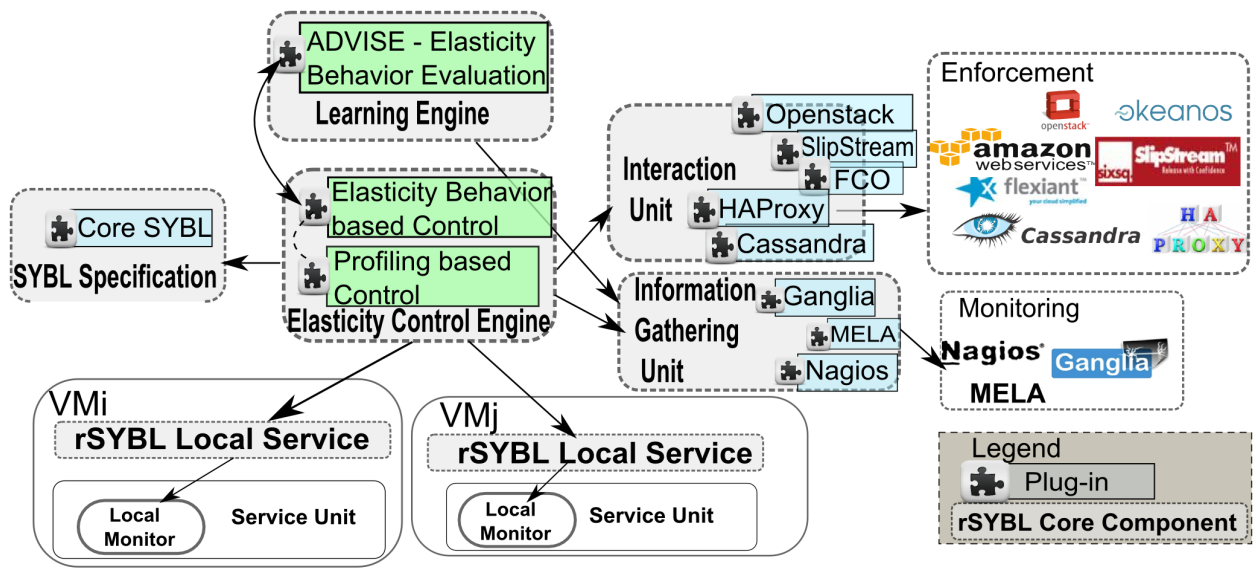

Fig. 9. ADVISE integration into rSYBL. 
Profiling-based control requires as input, per ECP, the expected effects obtained through manual or automated profiling. The effects which are available for this case are forecasts for expected metric values after finishing enforcing the respective ECP, and do not consider relationships among various SPs. The ECPs are enforced through the rSYBL Interaction Unit, which interacts with both cloud providerspecific APIs and with application specific control mechanisms.

For generating control plans, we interpret the effect, in time, of ECPs in relation to expected behavior without enforcing the respective control process. In this sense, elasticity behavior-based control estimates current metric evolution using a polynomial fitting approach. We obtain the elasticity requirements to be violated for the case where we do not enforce $\operatorname{ECP}_{\xi}$ (Eq. (9)) by computing the integral of violated requirements over estimated polynomial metric evolutions. Equation (10) shows the computation method for violated elasticity requirements for the case of enforcing $\mathrm{ECP}_{\xi}$, denoted ViolatedReq $\left(\mathrm{ECP}_{\xi}\right)$, using ADVISE estimations. We compare the two estimations, and select the one with the least violated requirements.

$$
\begin{aligned}
& \text { ViolatedReq }\left(\operatorname{ECP}_{\xi}\right)=\int_{\text {current }}^{\text {current }+\lambda} \operatorname{ViolReq}\left(P\left(\mathrm{SP}_{i}, \operatorname{Metric}_{j}(x)\right)\right) d x, \\
& \text { ViolatedReq }\left(\mathrm{ECP}_{\xi}\right)=\int_{\text {current }}^{\text {current }+\lambda} \text { ViolReq }\left(\operatorname{ADVISE}\left(\mathrm{SP}_{i}, \operatorname{Metric}_{j}(x)\right)\right) d x .
\end{aligned}
$$

We design the controller in such a way, that when the current behavior cannot be accurately estimated, we are able to rollback to profiling-based control. In this way, the enforced ECPs are not restricted solely to information obtained via the predeployment phase, instead the ADVISE behavior estimation is continuously refined improving the knowledge base and learning new behavior points representative for each ECP. In other words, when the closest estimated centroids are farther than a distance dist, empirically defined, the current decision making algorithm rolls back to the initial decision-making algorithm, as described in Ref. 8 .

\section{Experiments}

In this section, we provide an evaluation of the ADVISE framework, ${ }^{\mathrm{e}}$ focusing on the clustering-based behavior estimation process to determine the effectiveness of our approach as ADVISE can be used in both service profiling/pre-deployment or during runtime, for various service types, whenever monitoring information and enforced ECPs are available. As described in Sec. 2, ADVISE collects the following heterogeneous types of information, from plugins which we have developed, to populate the elasticity dependency graph: (i) Cloud service structural information, from TOSCA service descriptions; (ii) infrastructure and application performance

eCode, detailed descriptions and more charts at http://tuwiendsg.github.io/ADVISE. 


\section{G. Copil et al.}

information, from both JCatascopia ${ }^{10}$ and MELA ${ }^{11}$ monitoring systems; and (iii) elasticity information, regarding ECPs from the $\mathrm{rSYBL}^{8}$ elasticity controller.

Our evaluation is divided into two phases: (i) ADVISE framework evaluation; and (ii) ADVISE-enabled rSYBL evaluation. To evaluate the functionality of the ADVISE framework, we established a testbed on the Flexiant public cloud comprised of three cloud services originating from different service domains featuring distinct structural and behavior requirements. On the selected cloud services, we first, enforce ECPs exposed by their respected SPs randomly, and then study, at runtime, their behavior at multiple levels of the cloud service. It must be noted, that we did not configure rSYBL as a rational controller, since we are interested in estimating the elasticity behavior for all SPs as a result of enforcing both good and bad elasticity control decisions. For the second phase, we established a testbed on an OpenStack private cloud, with rSYBL elasticity control for a cloud service. We evaluate how ADVISE affects rSYBL elasticity control on various workloads.

\subsection{Experimental cloud services}

The first cloud service is a three-tier web application providing video streaming services to online users, comprised of: (i) an HAProxy Load Balancer which distributes client requests across multiple application servers; (ii) an Application Server Tier, where each application server is an Apache Tomcat server exposing the video streaming web service; and (iii) a Cassandra NoSQL Distributed Data Storage Backend from where the necessary video content is retrieved. The database backend initially holds $2 \mathrm{~GB}$ of data while at the end of the experiment the size approaches 6 GB. To stress this cloud service, we generate client requests under a fixed request rate, though the load is not stable and depends on the type of the requests (e.g. download video) and the size of the requested video, as shown in the workload pattern in Fig. 10(a).

The second cloud service in our evaluation is a Machine-to-Machine (M2M) DaaS which processes information originating from several different types of remote data sensors (e.g. temperature, atmospheric pressure, or pollution). Specifically, the M2M DaaS is comprised of an Event Processing Service Topology and a Data End Service Topology. Each service topology consists of two service units, one with a processing goal, and the other acting as the balancer/controller. To stress this cloud service, we generate random sensor event information (see Fig. 10(b)) which is processed by the Event Processing Service Topology, and stored/retrieved from the Data End Service Topology.

The third cloud service showcased is a two-tier OLTP service deployed as an online business directory hosting 7503 local business listings and their products. ${ }^{f}$ The topology of this service is comprised of a Document Store Controller,

${ }^{\mathrm{f}}$ Our dataset is synthetic, created from real data and workload patterns from www. finditcyprus.com. 

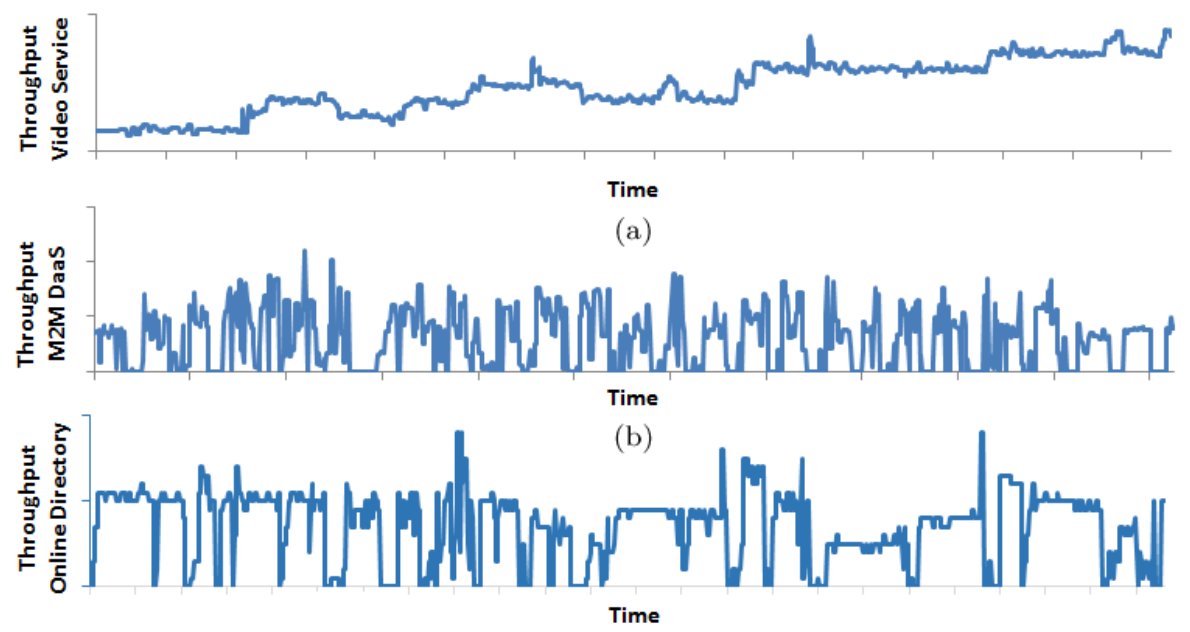

(c)

Fig. 10. Workload applied on the three services.

under a public domain, distributing client requests (i.e. create new listing, get directions to Restaurant $X$ ) to a Document Store Nodes, forming a Distributed Document Store which is a Couchbase database backend. Specifically, the database backend is a distributed, shared-nothing NoSQL (JSON-like) document store, optimized for interactive web applications, incorporating in its core application logic allowing developers to prepare and expose to their users queries as light-weight map/reduce functions (i.e. top- $k$ breweries in town Nicosia, etc.). We stress this service by generating client requests under a variable read-heavy request rate, mimicking a real online directory's behavior, as depicted in Fig. 10(c) (writes occur only when adding a new listing or updating an existing one and constitute less than $10 \%$ of the load). Tables 1 and 2 list the ECPs associated to each SP and the monitoring metrics analyzed for the three cloud services respectively.

\subsection{Cloud service elasticity behavior evaluation}

\subsubsection{ECP temporal effect}

ADVISE computes, as shown in Table 3, the average time required for an ECP to be completed, and returns also a standard deviation which gives the degree of confidence with regard to this estimation. This application-specific information is of high importance and affects the decision-making process of the elasticity controller since it is an indicator of the grace period which it should await until effects of the resizing actions are noticeable. Thus, it can define the time granularity of which resizing actions should be taken into consideration. For example, we observe that the process of reconfiguring and removing an instance from the video service's storage backend requires an average time interval of $160 \mathrm{~s}$ which is mainly due the 


\section{G. Copil et al.}

Table 1. Elasticity control processes available for the cloud services.

\begin{tabular}{|c|c|c|}
\hline $\begin{array}{l}\text { Cloud } \\
\text { Service }\end{array}$ & ECP ID & Action Sequence \\
\hline \multirow[t]{4}{*}{ Video Service } & $\mathrm{ECP}_{1}$ & $\begin{array}{l}\text { Scale In Application Server Tier: (i) stop the video streaming } \\
\text { service, (ii) remove instance from HAProxy, (iii) restart } \\
\text { HAProxy, (iv) stop JCatascopia Monitoring Agent, (v) delete } \\
\text { VM }\end{array}$ \\
\hline & $\mathrm{ECP}_{2}$ & $\begin{array}{l}\text { Scale Out Application Server Tier: (i) create new network } \\
\text { interface, (ii) instantiate new VM, (iii) deploy and configure } \\
\text { video streaming service, (iv) deploy and start JCatascopia } \\
\text { Monitoring Agent, (v) add VM IP to HAProxy, (vi) restart } \\
\text { HAProxy }\end{array}$ \\
\hline & $\mathrm{ECP}_{3}$ & $\begin{array}{l}\text { Scale In Distributed Video Storage Backend: (i) select VM to } \\
\text { remove, (ii) decommission instance data to other nodes, } \\
\text { (iii) stop JCatascopia Monitoring Agent, (iv) delete VM }\end{array}$ \\
\hline & $\mathrm{ECP}_{4}$ & $\begin{array}{l}\text { Scale Out Distributed Video Storage Backend: (i) create new } \\
\text { network interface, (ii) instantiate new VM, (iii) deploy and } \\
\text { configure Cassandra (e.g. assign token to node), (iv) deploy } \\
\text { and start JCatascopia Monitoring Agent, (v) start Cassandra }\end{array}$ \\
\hline \multirow[t]{4}{*}{ M2M DaaS } & $\mathrm{ECP}_{5}$ & $\begin{array}{l}\text { Scale In Event Processing Service Unit: (i) remove service from } \\
\text { HAProxy, (ii) restart HAProxy, (iii) remove recursively VM }\end{array}$ \\
\hline & $\mathrm{ECP}_{6}$ & $\begin{array}{l}\text { Scale Out Event Processing Service Unit: (i) create new network } \\
\text { interface, (ii) create new VM, (iii) add service IP to HAProxy }\end{array}$ \\
\hline & $\mathrm{ECP}_{7}$ & $\begin{array}{l}\text { Scale In Data Node Service Unit: (i) decommission node (copy } \\
\text { data from VM to be removed), (ii) remove recursively VM }\end{array}$ \\
\hline & $\mathrm{ECP}_{8}$ & $\begin{array}{l}\text { Scale Out Data Node Service Unit: (i) create new network } \\
\text { interface, (ii) create VM, (iii) set ports, (iv) assign token to } \\
\text { node, (v) set cluster controller, (vi) start Cassandra }\end{array}$ \\
\hline \multirow[t]{2}{*}{$\begin{array}{l}\text { Online } \\
\text { Directory }\end{array}$} & $\mathrm{ECP}_{9}$ & $\begin{array}{l}\text { Scale In Distributed Document Store: (i) select Couchbase-server } \\
\text { to remove, (ii) decommission node from Couchbase cluster, } \\
\text { (iii) rebalance cluster data, (iv) remove VM }\end{array}$ \\
\hline & $\mathrm{ECP}_{10}$ & $\begin{array}{l}\text { Scale Out Distributed Document Store: (i) create new network } \\
\text { interface, (ii) instantiate VM, (iii) configure interfaces, ports, } \\
\text { and Couchbase-server (iv) start Couchbase-server, (v) join } \\
\text { Couchbase cluster, (vi) rebalance cluster }\end{array}$ \\
\hline
\end{tabular}

Table 2. Elasticity metrics per cloud service for different service parts.

\begin{tabular}{|c|c|c|}
\hline $\begin{array}{l}\text { Cloud } \\
\text { Service }\end{array}$ & SP Name & Metrics \\
\hline Video Service & $\begin{array}{l}\text { Application Server } \\
\text { Tier } \\
\text { Distributed Video } \\
\text { Storage Backend }\end{array}$ & $\begin{array}{l}\text { Cost, busy thread number, request throughput } \\
\text { Cost, CPU usage, memory usage, query latency }\end{array}$ \\
\hline M2M DaaS & $\begin{array}{l}\text { Cloud Service } \\
\text { Event Processing } \\
\text { Service Topology } \\
\text { Data End Service } \\
\text { Topology }\end{array}$ & $\begin{array}{l}\text { Cost per client per hour } \\
\text { Cost, response time, throughput, number of clients } \\
\text { Cost, latency, CPU usage }\end{array}$ \\
\hline $\begin{array}{l}\text { Online } \\
\text { Directory }\end{array}$ & $\begin{array}{l}\text { Document Store } \\
\text { Controller } \\
\text { Distributed Store } \\
\text { Node }\end{array}$ & $\begin{array}{l}\text { Cost, request rate, active sessions, error rate, CPU } \\
\text { usage, network I/O } \\
\text { Cost, throughput, cache miss rate, disk I/O, memory } \\
\text { usage, CPU usage, query response time }\end{array}$ \\
\hline
\end{tabular}


Table 3. Elasticity control processes time statistics.

\begin{tabular}{lccc}
\hline & ECP & Standard Deviation & Average ECP Time (s) \\
\hline Video Service & ECP1 & 0.06 & 90 \\
& ECP2 & 0.12 & 25 \\
& ECP3 & 0.13 & 160 \\
& ECP4 & 0.11 & 30 \\
M2M Service & ECP5 & 0.34 & 45 \\
& ECP6 & 0.16 & 20 \\
& ECP7 & 0.11 & 70 \\
Online Directory & ECP8 & 0.14 & 20 \\
& ECP9 & 0.29 & 110 \\
& ECP10 & 0.12 & 25 \\
\hline
\end{tabular}

time required to receive and store data from other nodes of the ring. If decisions are taken in smaller intervals, the effects of the previous action will not be part of the current decision process and may cause cascading ping-pong effects where a Scale In ECP followed by a slight increase in metric utilization (in the grace period) causes a false Scale Out ECP.

\subsubsection{Online video streaming service - Elasticity behavior estimation}

Figure 11 depicts both the observed and the estimated behavior for the Video Service Application Server Tier when ECPs of type $\mathrm{ECP}_{1}$ (remove application server) are enforced. At first, we observe that the average request throughput per application server is decreasing. This is due to two possible cases: (i) The video storage backend is under-provisioned and cannot satisfy the current number of requests which, in turn, results in requests being queued; (ii) there is a sudden drop in client requests which indicates that the application servers are not utilized efficiently. For an elasticity controller driven by simple "if-then-else" policies for applicationspecific metrics (e.g. request throughput) there is no apparent way in determining the case in hand and it will act upon metric violations without considering if an ECP will indeed improve QoS or cost. From Fig. 11, we observe that after the Scale In ECP occurs, the average request throughput and busy thread number rises which denotes that this behavior corresponds to the second case where resources are now efficiently utilized. ADVISE revealed an insightful correlation between two metrics to consider in the decision process.

Similarly, in Fig. 12, we depict both the observed and the estimated behavior for the Distributed Video Storage Backend when a Scale Out ECP is enforced (add Cassandra Node to ring) due to high CPU utilization. We observe that after the Scale Out ECP is enforced, the actual CPU utilization decreases to a normal value as also indicated by the estimation.

Analyzing the estimations made for this service (i.e. Figs. 11 and 12), we conclude that the estimations provided by ADVISE successfully follow the actual behavior pattern and that, as time intervenes, the curves tend to converge. 
G. Copil et al.

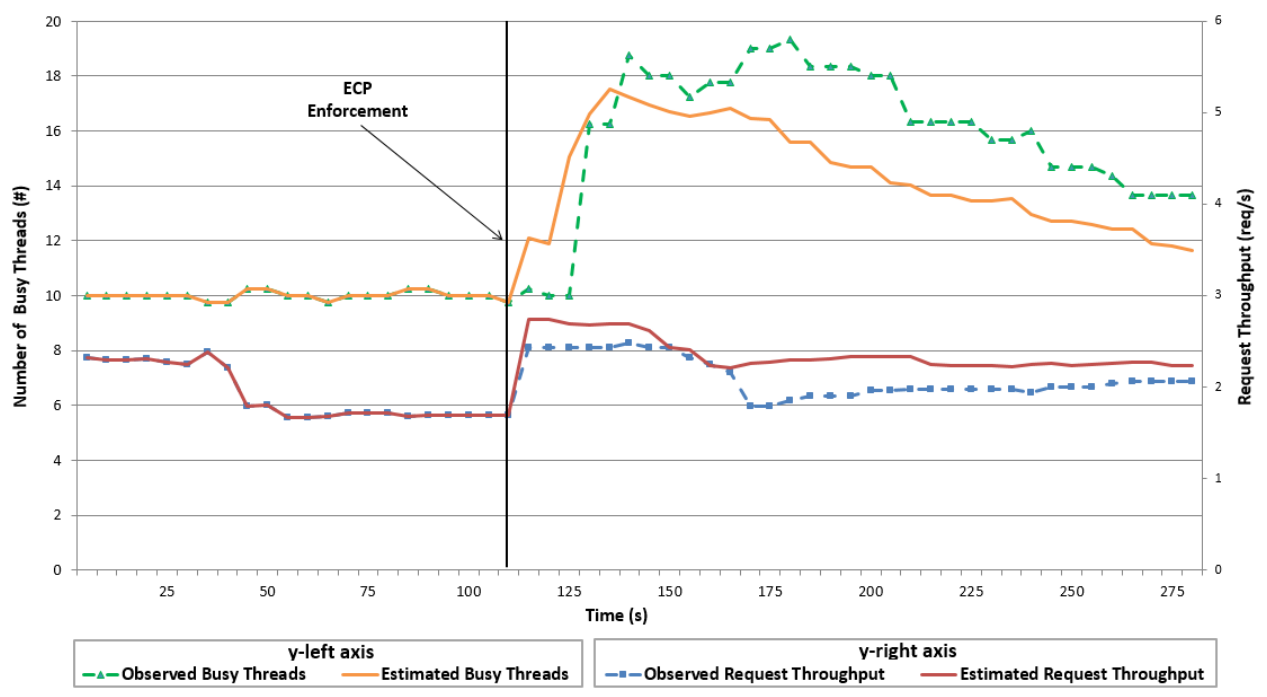

Fig. 11. Effect of $\mathrm{ECP}_{1}$ on the application server tier.

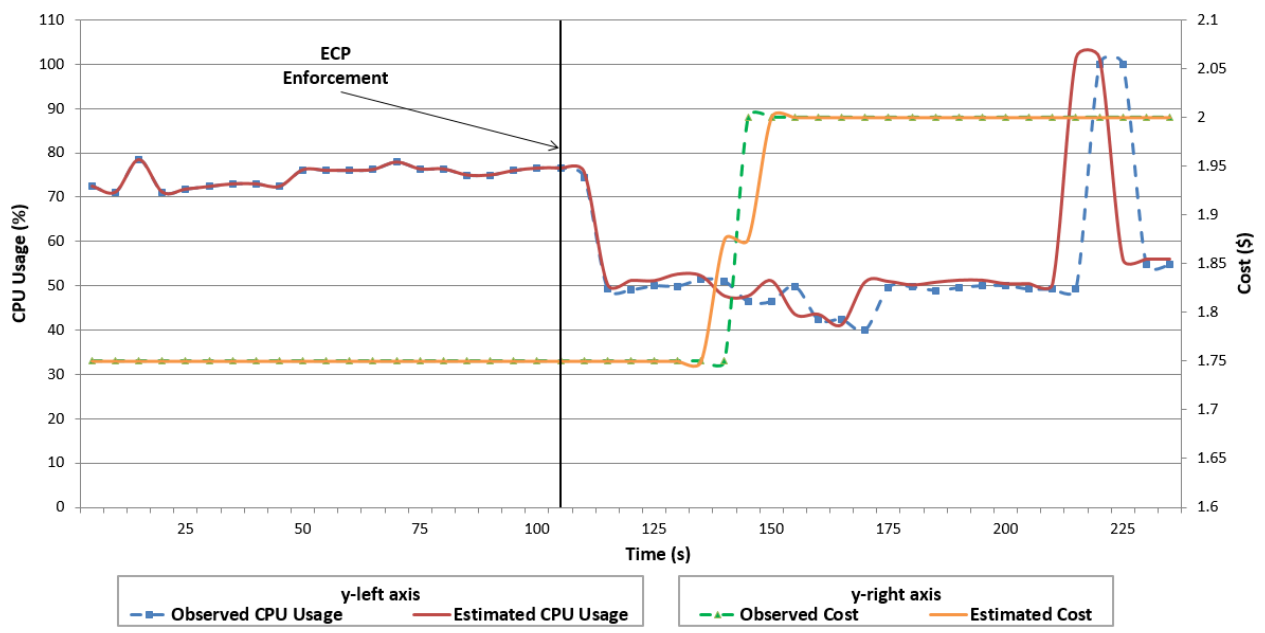

Fig. 12. Effect of $\mathrm{ECP}_{4}$ on the entire video streaming service.

\subsubsection{M2M DaaS - Elasticity behavior estimation}

Figure 13 showcases how an ECP targeting a service unit affects the entire cloud service. The Cost/Client/h is a complex metric (see Table 2) which depicts how profitable is the service deployment in comparison to the current number of users. Although Cost/Client/h is not accurately estimated, due to the high fluctuation in number of clients, our approach approximates how the cloud service would behave in terms of expected metric fluctuations. This information is important for elasticity 

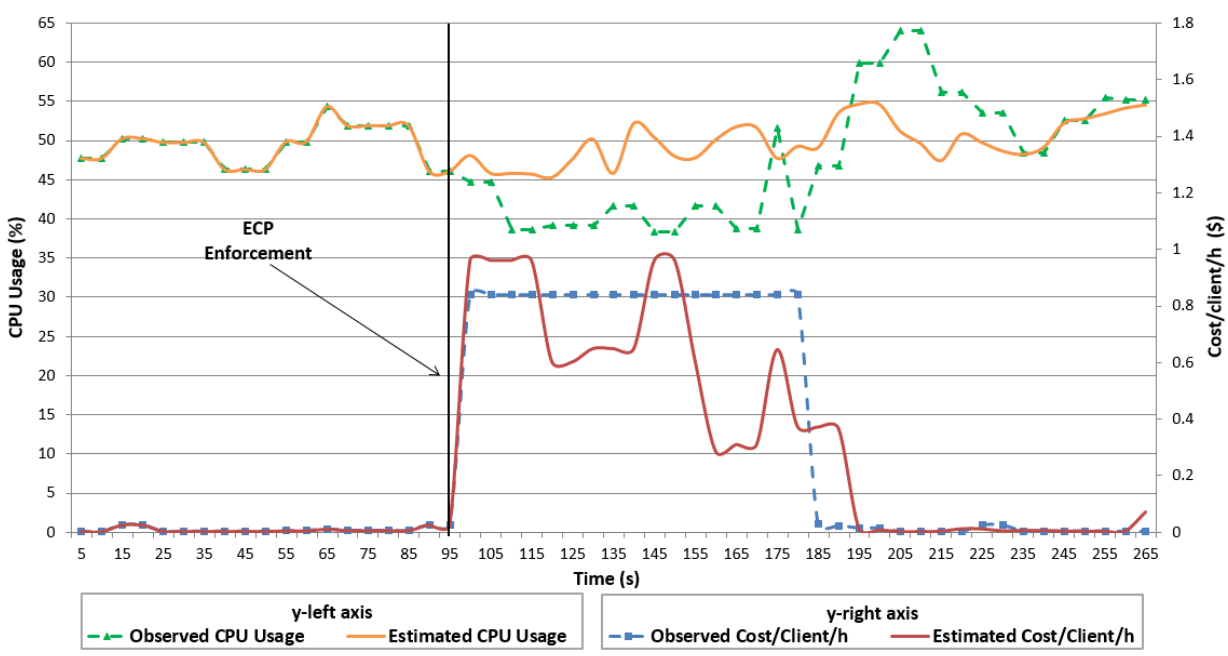

Fig. 13. Effect of $\mathrm{ECP}_{7}$ on M2M DaaS.

controllers to improve their decisions when enforcing ECPs by knowing how the Cost/Client/h for the entire cloud service would be affected. Although CPU usage is not estimated perfectly, since it is a highly oscillating metric, and it depends on the CPU usage at each service unit level, knowing the baseline of this metric can also help in deciding whether this ECP is appropriate (e.g. for some applications CPU usage above $90 \%$ for a period of time might be inadmissible). Figure 14 shows estimations of behavior for the Event Processing Service Topology, when a Scale

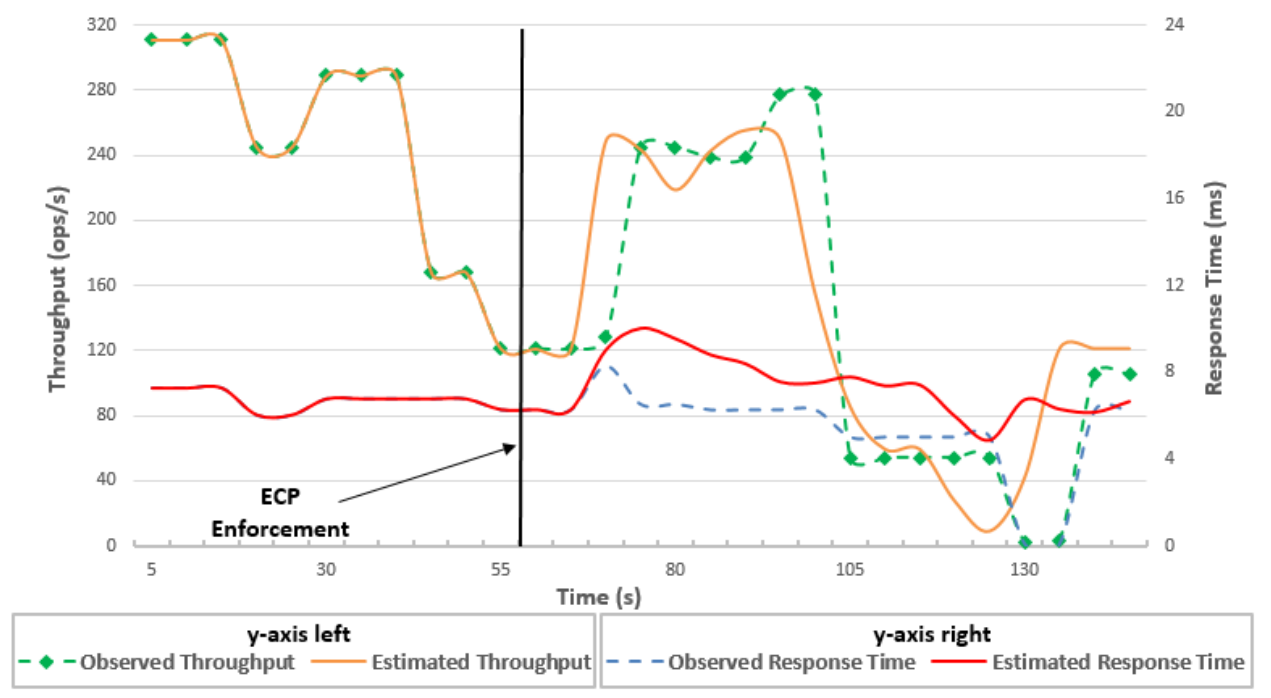

Fig. 14. Effect of $\mathrm{ECP}_{6}$ on the event processing service topology. 


\section{G. Copil et al.}

Out ECP occurs on the Event Processing Service Unit. Although the throughput is accurately estimated with a slight lag, response time is estimated with a slightly larger error due to the fact that a down peak is not estimated, as not being part of the usual behavior for the current SP.

ADVISE can estimate the effect of an ECP of a SP, on a different SP, even if apparently unrelated and therefore provide, multi-grain elasticity behavior evaluation. Figure 15 depicts an estimation on how the Data Controller Service Unit is impacted by the data transferred at the enforcement of $\mathrm{ECP}_{8}$. In this case, the controller CPU usage initially, as one would expect, decreases since the new node will offload other nodes, however, effort is required in transferring data to the new node which rises utilization due to the fact that reconfigurations are also necessary on the controller, following a slight decrease and then stabilization. Therefore, even in circumstances of random workload, ADVISE can give useful insights on how different SPs behave when enforcing ECPs exposed by other SPs which, again, elasticity controllers have no knowledge of.

\subsubsection{Online directory - Elasticity behavior estimation}

Figure 16 depicts the observed and estimated throughput and CPU usage measured at the Document Store Controller after a Scale Out ECP is enforced. The Document Store Controller is a document store node itself, however, it features additional functionality: It supervises (meta-)data migration when the cluster is re-balanced as in the case of a Scale In/Out ECP enforcement. While other nodes continue to accept client requests when an ECP is enforced, the Document Store Controller prioritizes the supervisioning of data movement and thus, ceases to process client requests. This is evident in Fig. 16, where we observe that when the cluster is rebalanced, throughput drops to zero while CPU usage does not decrease, as one would expect,

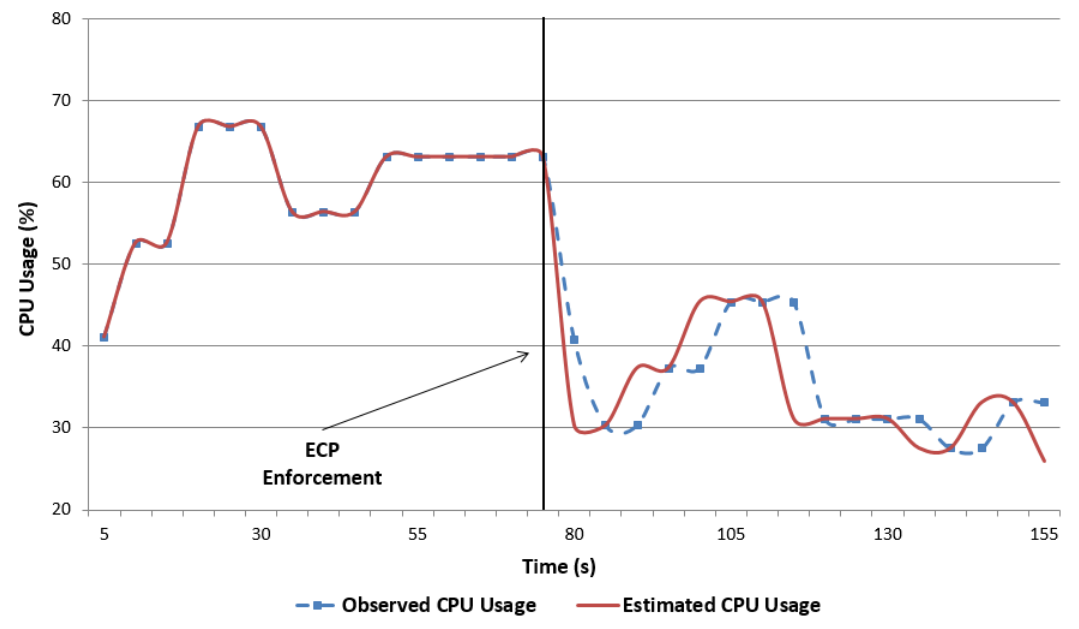

Fig. 15. Effect of $\mathrm{ECP}_{8}$ on the data controller service unit. 


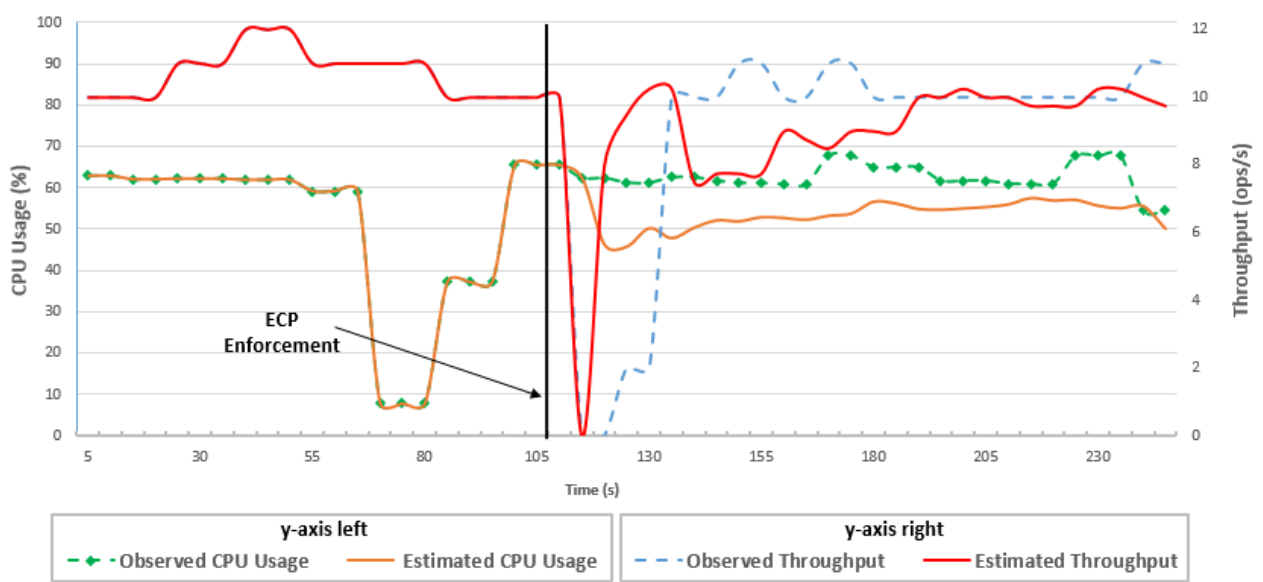

Fig. 16. Effect of $\mathrm{ECP}_{10}$ on the document store controller.

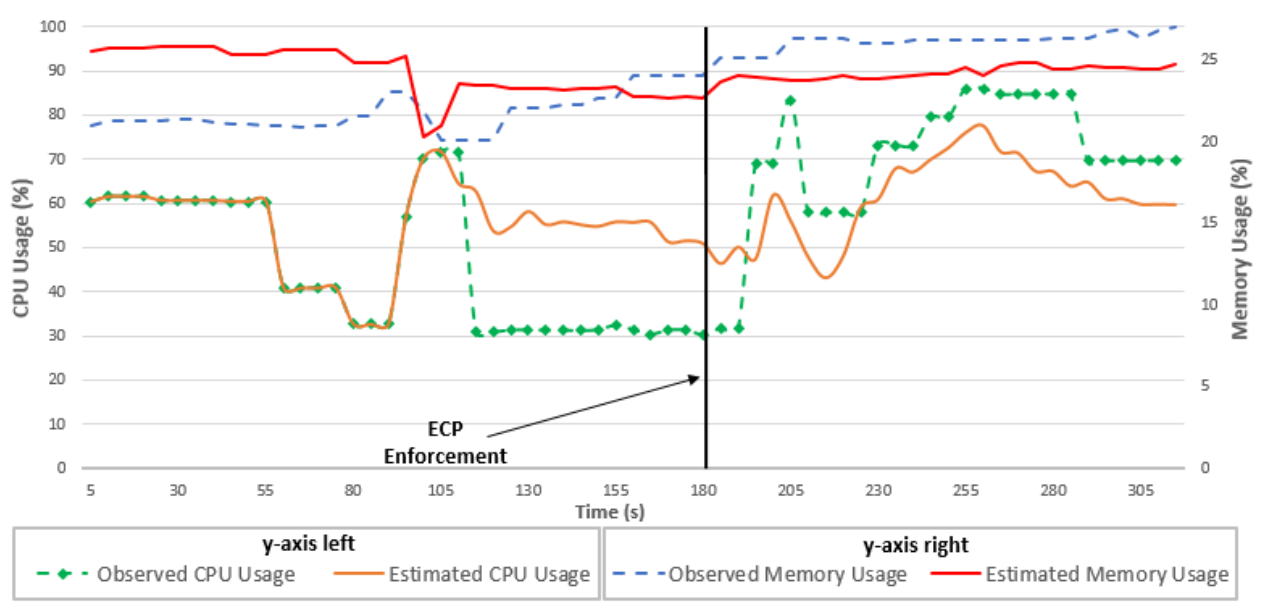

Fig. 17. Effect of $\mathrm{ECP}_{9}$ on the document store node.

until after rebalancing is complete. In turn, Fig. 17 depicts the effects of a Scale In $\mathrm{ECP}$ on one of the document store nodes. ADVISE identifies the increase in memory utilization as the node receives part of the load from the decommissioned node, while the estimation for CPU utilization follows the observed oscillating trend.

On the other hand, Fig. 18 depicts both CPU usage and cost of a document store node before and after a Scale Out ECP. We observe that before the ECP enforcement the ADVISE estimation follows the observed values, however, after the ECP enforcement, the provided estimation slightly deviates from the observed CPU utilization before converging again. The reason behind this slight deviation is due to, an out of the ordinary, large data movement, not evident in most Scale Out ECPs, where the whole dataset must be replicated since the Scale Out ECP 


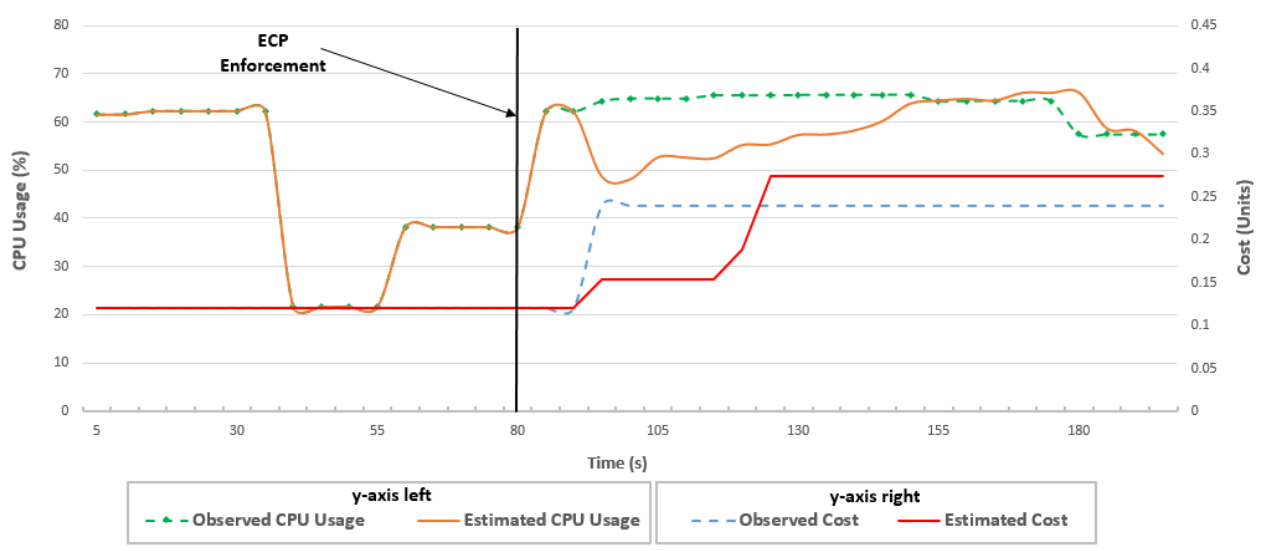

Fig. 18. Effect of $\mathrm{ECP}_{10}$ on the data node.

occurs immediately after a series of multiple Scale In ECPs which left the cluster with only two instances.

\subsubsection{Quality of results}

ADVISE is able to estimate, in time, the elasticity behavior of different SPs by considering the correlations amongst metrics and the ECPs which are enforced. To evaluate the quality of our results, we have considered the fact that existing tools do not produce continuous-time estimations. Thus, we evaluate ADVISE by computing the variance Var and standard deviation StdDev (Eq. (11)), over 100 estimations as the result differs little afterwise

$$
\begin{aligned}
\operatorname{Var}_{\text {metric }} & =\frac{\sum \frac{\sum_{i=\left[0, \text { rts }_{\text {size }}\right]}\left(\text { estMetric }_{i}-\text { obsMetric }_{i}\right)^{2}}{\text { rts }_{\text {size }}}}{\text { nbEstimations }-1} \text { StdDev }_{\text {metric }} \\
& =\sqrt{\operatorname{Var}_{\text {metric }_{i}}} .
\end{aligned}
$$

Table 4 presents the accuracy of our results. When comparing the three services, the Video Service achieves a higher accuracy (smaller standard deviation), since the imposed workload is considerably stable. Focusing on the M2M DaaS estimation accuracy, we observe that it depends on the granularity at which the estimation is calculated, and on the ECP. Moreover, the standard deviation depends on the metrics monitored for the different parts of the cloud service. For instance, in the case of the M2M Service, the number of clients metric can be hardly predicted, since we have sensors sending error or alarm-related information. This is evident for the Event Processing Service Topology, where the maximum variance for the number of clients is 4.9 .

Overall, even in random cloud service load situations, the ADVISE framework analyzes and provides estimations for elasticity controllers, allowing them 
Table 4. ECPs effect estimation quality statistics.

\begin{tabular}{llcccc}
\hline $\begin{array}{l}\text { Cloud } \\
\text { Service }\end{array}$ & \multicolumn{1}{c}{$\begin{array}{c}\text { Observed Cloud } \\
\text { Service Part }\end{array}$} & $\begin{array}{c}\text { Elasticity } \\
\text { Control } \\
\text { Process }\end{array}$ & $\begin{array}{c}\text { Average } \\
\text { Standard } \\
\text { Deviation }\end{array}$ & $\begin{array}{c}\text { Maximum } \\
\text { Variance }\end{array}$ & $\begin{array}{c}\text { Minimum } \\
\text { Variance }\end{array}$ \\
\hline Video & Video Service & $\mathrm{ECP}_{3}$ & 0.23 & 0.09 & 0.03 \\
Service & & $\mathrm{ECP}_{4}$ & 0.61 & 0.99 & 0.23 \\
& Distributed Video Storage & $\mathrm{ECP}_{3}$ & 0.28 & 0.14 & 0.034 \\
& Backend & $\mathrm{ECP}_{4}$ & 0.2 & 0.042 & 0.04 \\
& Application Server & $\mathrm{ECP}_{1}$ & 0.43 & 0.4 & 0.06 \\
& & $\mathrm{ECP}_{2}$ & 0.31 & 0.47 & 0.01 \\
M2M & Cloud Service & $\mathrm{ECP}_{5}$ & 0.9 & 6.65 & 0.24 \\
Service & Data End Service Topology & $\mathrm{ECP}_{5}$ & 0.23 & 0.35 & $7.44 \mathrm{E}-05$ \\
& Event Processing Service & $\mathrm{ECP}_{7}$ & 1.1 & 4.9 & 0.046 \\
& Topology & $\mathrm{ECP}_{8}$ & 0.76 & 2.46 & 0.027 \\
& Data Controller Service & $\mathrm{ECP}_{6}$ & 0.12 & 0.25 & 0 \\
& Unit & $\mathrm{ECP}_{8}$ & 0.22 & 0.41 & 0 \\
& Data Node Service Unit & $\mathrm{ECP}_{5}$ & 0.572 & 0.68 & 0.32 \\
& & $\mathrm{ECP}_{6}$ & 0.573 & 1.4 & 0.07 \\
& Event Processing Service & $\mathrm{ECP}_{7}$ & 1.08 & 3.59 & 0.11 \\
& Unit & $\mathrm{ECP}_{8}$ & 0.77 & 1.9 & 0.14 \\
Online & Document Store Node & $\mathrm{ECP}_{9}$ & 0.19 & 0.05 & 0.29 \\
Directory & Document Store Node & $\mathrm{ECP}_{10}$ & 0.14 & 0.005 & 0.18 \\
& Document Store Controller & $\mathrm{ECP}_{10}$ & 0.13 & 0.023 & 0.38 \\
\hline
\end{tabular}

to improve the quality of control decisions, with regard to the evolution of monitored metrics at the different cloud service levels. Moreover, these estimations are delivered together with the confidence of the estimation, given by the distance from current behavior point to the estimated behavior point. Without this kind of estimation, elasticity controllers would need to use VM-level profiling information, while having to control complex cloud services. This information, for each SP, is valuable for controlling elasticity of complex cloud services, which expose complex control mechanisms.

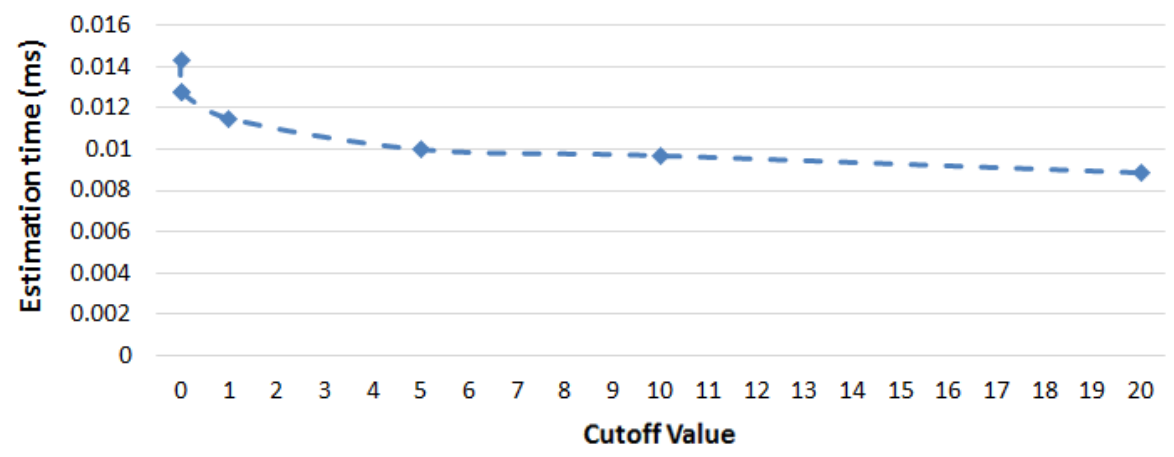

Fig. 19. $\mathrm{ECP}_{5}$ estimation time under different cutoff values. 
G. Copil et al.

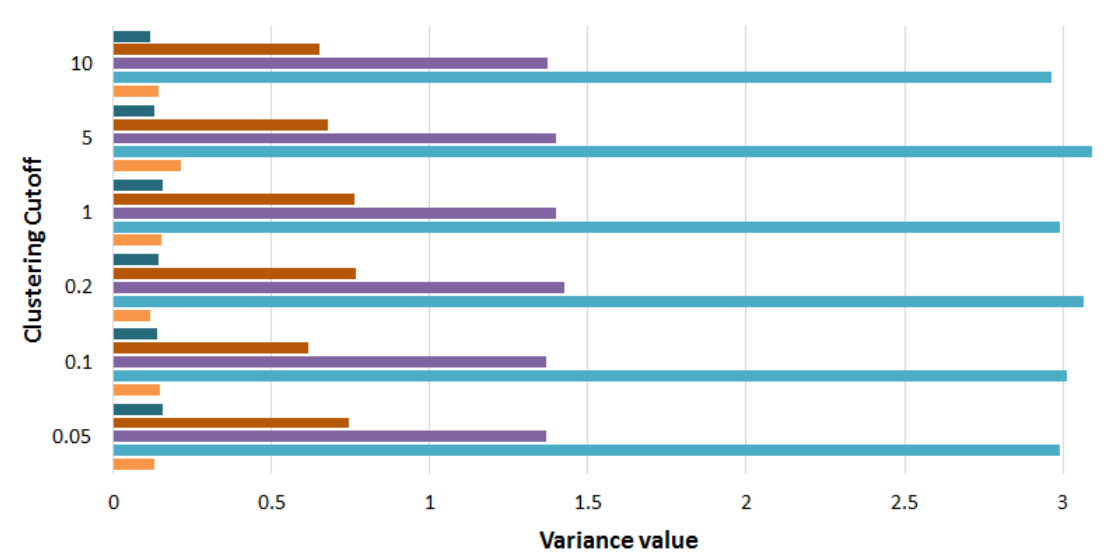

Number of VMs variance Active connections variance $\square$ Throughput variance $\quad$ Response time variance

Fig. 20. Estimation variance for $\mathrm{ECP}_{5}$ under different cutoff values.

\subsubsection{Sensitivity of results}

For analyzing the sensitivity of our results, we evaluated how our empirically determined parameters (e.g. clustering offset) affect the variance of the estimation in regards to the observed behavior. Figure 20 concludes that our results are very little affected by the choice of the offset.

When analyzing the impact that the choice of the offset has over time (Fig. 22(b)), we can see that very small offset values reflect in a considerable increase in the estimation time. This is why, the offset was chosen at 0.2 , as a tradeoff between estimation time and estimation quality.

\section{3. $r S Y B L$ elasticity control enhanced with ADVISE estimations}

As described in Sec. 4, we integrate the ADVISE behavior estimation into the rSYBL elasticity controller, which controls elasticity at multiple levels of abstraction. $^{8}$

We use the M2M DaaS service with fixed Data End Topology, while controlling Event Processing Topology using $\mathrm{ECP}_{5}$ (i.e. Scale In) and $\mathrm{ECP}_{6}$ (i.e. Scale Out) elasticity control processes on an OpenStack private cloud, with the following SYBL $^{9}$ elasticity requirements:

- EventProcessingTopology - Co1:CONSTRAINT responseTime $<100 \mathrm{~ms}$.

- EventProcessing Topology — St1:STRATEGY CASE responseTime < 12 AND throughput < 100: minimize (cost).

We compare the elasticity control performed by ADVISE-enabled rSYBL decision making and respectively the profiling-based decision making. We apply a stepwise workload in order to observe controller's behavior under different circumstances. Figure 21 shows at (a) the outcome of controlling the service with 


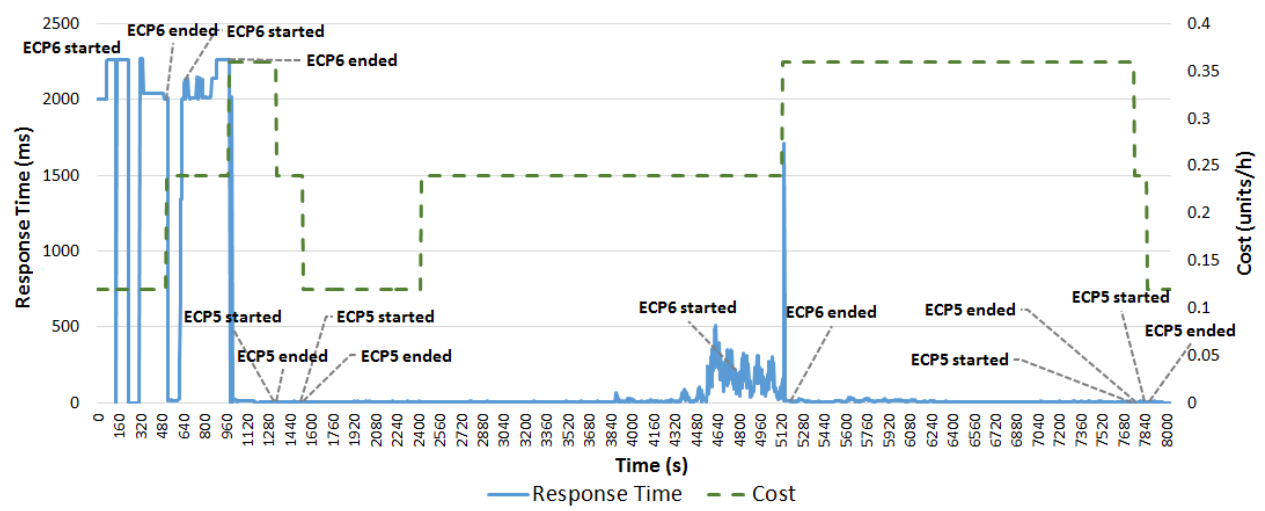

(a)

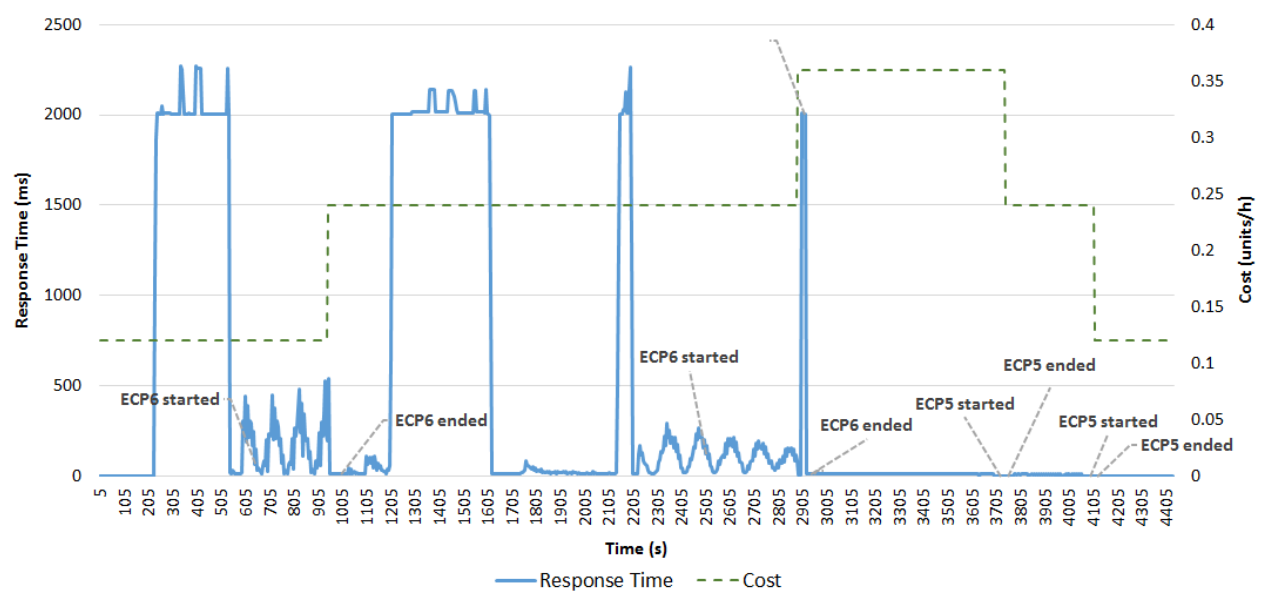

(b)

Fig. 21. Event Processing Topology control. (a) rSYBL with ADVISE knowledge, (b) rSYBL without ADVISE.

ADVISE-enabled rSYBL and at (b) the outcome of controlling the service considering profiling information, consisting of how much the metrics would be affected by enforcing an ECP. At first, we observe that ADVISE-based control provides the elasticity controller with a better elasticity behavior understanding, even in cases where metric values exceed their expected values (i.e. in this case due to a memory bottleneck). However, it accomplishes this with a bigger cost, as one would expect, since it is using more resources to fulfill the requirements (e.g. in this case ADVISE-enabled controller achieves a cost $27.5 \%$ higher). In contrast with this, the profiling based elasticity controller is not able to find appropriate ECPs in unexpected situations, when the value observed exceeds its possibilities of controlling the metric, as known from profiling information (e.g. if the response time is expected to decrease with $200 \mathrm{~ms}$ whenever ECP6 is enforced, while current metric values 


\section{G. Copil et al.}

are $2000 \mathrm{~ms}$ ). Moreover, the ADVISE-based control considers the following interval when analyzing the expected behavior, not only the final metric values. Thus, whenever enforcing an ECP results in comparable requirements violation over the whole period as for the case of no ECP enforcement, the controller chooses not to take any action. When applying a steady/fix workload, depending on the formulated requirements, some controllers can reach continuous control oscillations. This was also the case for rSYBL with profiling information, as we can see from Fig. 22(a). Due to the continuous effects which are being used by rSYBL with ADVISE, on the same workload, and the same elasticity requirements, this "ping-pong" effect is avoided (see Fig. 22(b)), since the controller knows that the enforcement $\mathrm{ECP}_{5}$ will imply the overall increase of the response time, after a time period.

Using ADVISE in elasticity control can also avoid situations where various control processes are enforced without understanding their outcome. For instance,

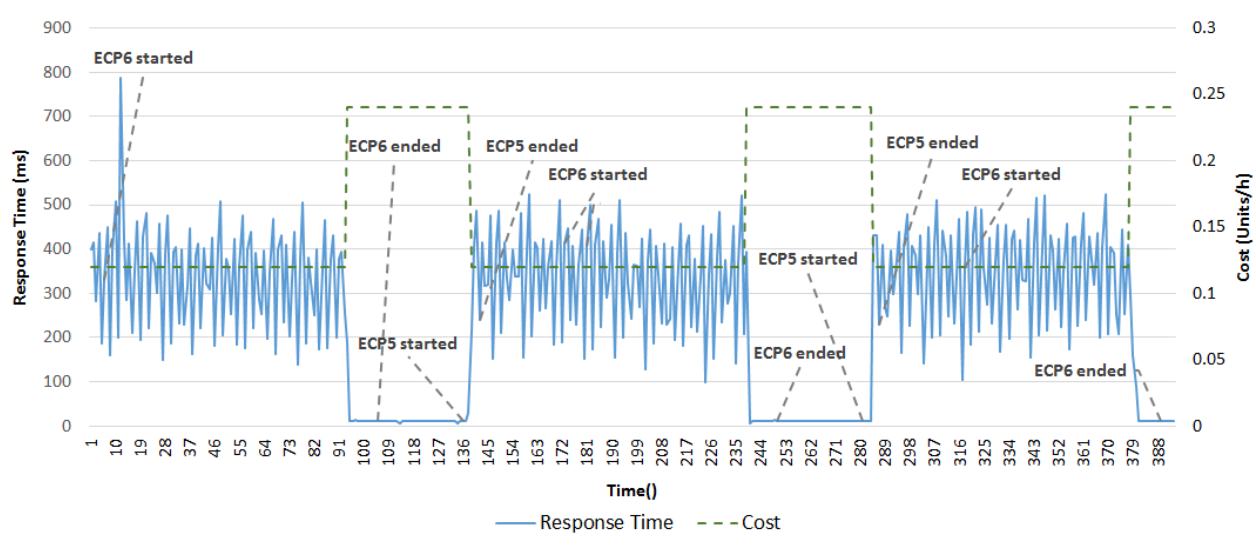

(a)

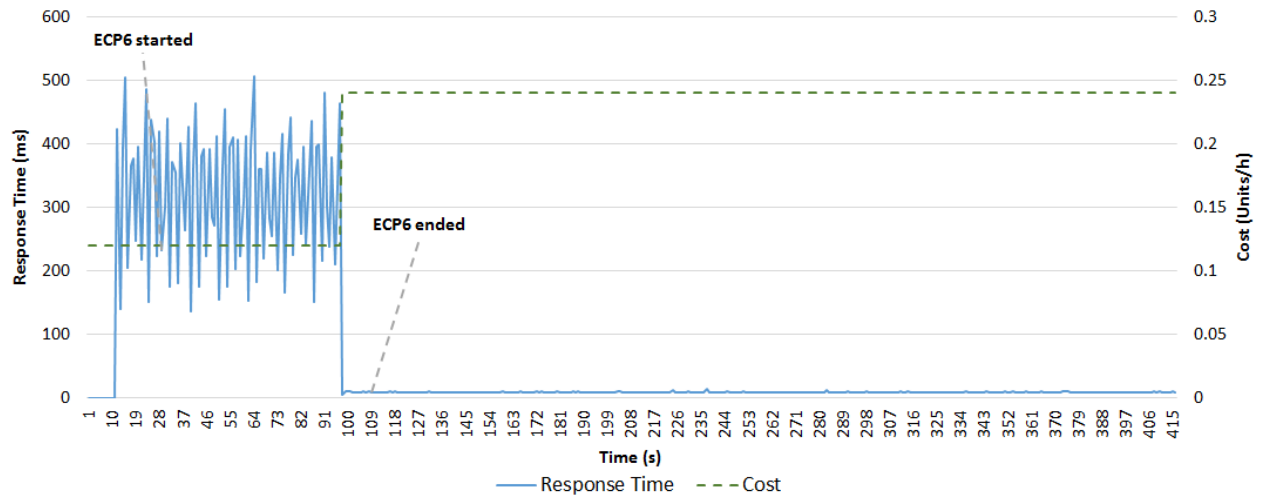

(b)

Fig. 22. Ping-pong effect. (a) "Ping-pong" effect for steady workload with rSYBL, (b) no "pingpong" when using ADVISE. 
enforcing Scale Out when response time satisfies a condition does not always result in response time decrease. Moreover, in some cases, where the service is not truly elastic, enforcing ECPs considering expected discrete effects would only cause increase in costs. With ADVISE and SYBL, the control processes are enforced only when, considering current and previous behavior, the ECP would help fulfilling SYBL requirements (e.g. increase throughput, minimize response time).

\section{Related Work}

In our previous work, we focused on modeling current and previous behavior with the concepts of elasticity space and pathway, ${ }^{11}$ where we utilize different algorithms to determine enforcement times in observed service behavior (e.g. with change-point detection), but without modeling expected behavior of different service parts, in time. Verma et al. ${ }^{5}$ study the impact of reconfiguration actions on system performance. They observe infrastructure level reconfiguration actions, with actions on live migration, and observe that the VM live migration is affected by the CPU usage of the source virtual machine, both in terms of the migration duration and application performance. The authors conclude with a list of recommendations on dynamic resource allocation. Kaviani et al. ${ }^{16}$ propose profiling as a service, to be offered to other cloud customers, trying to find tradeoffs between profiling accuracy, performance overhead, and costs incurred. Zhang et al. ${ }^{7}$ propose algorithms for performance tracking of dynamic cloud applications, predicting metrics values like throughput or response time.

Dean et $a .^{17}$ propose an approach for predicting running application performance anomalies, self-organizing maps for capturing emergent behavior and predicting unknown anomalies. Using unsupervised learning, this approach also identifies previously unknown anomalies/faults which may appear in the system (e.g. memory leaks, cpu leaks). For cloud service SLA violation prediction several solutions have been proposed, such as Refs. 18 and 19, which use statistical models (e.g. decision trees, artificial neural networks) or naive bayes classifiers, predicting when the SLA would be violated without focusing on the violation cause. LaCurts et $a l_{.}{ }^{20}$ propose Cicada, a framework which predicts network traffic for cloud applications, without making assumptions about application structure. The authors argue that cloud providers should offer predictive guarantees as a service, instead of bandwidth guarantees, which would also encapsulate application runtime changes. Similarly, ADVISE-enabled rSYBL can be used to guarantee or to sell as a service cloud service elasticity, with little specifications coming from the user.

Juve et al. ${ }^{21}$ propose a system which helps automating the provisioning process for cloud-based applications. They consider two application models, one workflow application and one data storage case, and show how for these cases the applications can be deployed and configured automatically. Li et al. ${ }^{22}$ propose CloudProphet framework, which uses resource events and dependencies among them for predicting web application performance on the cloud. Shen et al. ${ }^{6}$ propose the 


\section{G. Copil et al.}

CloudScale framework which uses resource prediction for automating resource allocation according to service level objectives (SLOs) with minimum cost. Based on resource allocation prediction, CloudScale uses predictive migration for solving scaling conflicts (i.e. there are not enough resources for accommodating scale-up requirements) and CPU voltage and frequency for saving energy with minimum SLOs impact. Compared with this research work, we construct our model considering multiple levels of metrics, depending on the application structure for which the behavior is learned. Moreover, the stress factors considered are also adapted to the application structure and the elasticity capabilities (i.e. action types) enabled for that application type.

Compared with presented research work, we focus not only on estimating the effect of an elasticity control process on the service part with which it is associated, but on different other parts of the cloud service. Moreover, we estimate and evaluate the elasticity behavior of different cloud service parts, in time, because we are not only interested in the effect after a predetermined period, but also with the pattern of the effect that the respective ECP introduces.

\section{Conclusions and Future Work}

It is important to understand elasticity behavior of complex cloud services due to different control processes, in order to support better elasticity provisioning. In this paper, we have presented a methodology for estimating cloud service elasticity behavior, and implemented it in our ADVISE framework. ADVISE is able to estimate the behavior of cloud service parts, in time, when enforcing various ECPs, by considering different types of information represented through the elasticity dependency graph. Experiments from three different cloud services, show that ADVISE framework is indeed able to advise elasticity controllers about cloud service behavior, contributing towards improving cloud service elasticity. We show how we integrate ADVISE with the rSYBL elasticity controller, ${ }^{8}$ and the decision mechanisms we refined in order to consider continuous ECP effects. We have shown the improvement that ADVISE brings to the rSYBL elasticity controller, and discussed the various decision types that ADVISE influences.

As future work, we will focus on further understanding how heterogeneous resources used in the control process would affect the cloud service elasticity behavior. Moreover, we are considering various aspects which are dynamic in a complex cloud service. For instance, communication patterns might change at runtime as a reaction of the service to incoming load properties (e.g. service units which are re-grouping into other topologies when the users interests, and load characteristics, change). Moreover, enforcing elasticity capabilities can also involve changes in service structure (e.g. adding another layer of balancing involves creating a sub-topology in an already existing topology). Focusing on these aspects, we are investigating how communication and structural dynamism affect the cloud service 
behavior, how these can be estimated, and finally, how can they be integrated in the ADVISE framework.

\section{Acknowledgments}

This work was supported by the European Commission in terms of the CELAR FP7 project (FP7-ICT-2011-8 \#317790).

\section{References}

1. 2014 State of the Cloud Survey, http://www.rightscale.com/blog/cloud-computingtrends-2014-state-cloud-survey.

2. S. Dustdar, Y. Guo, B. Satzger and H.-L. Truong, Principles of elastic processes, IEEE Internet Comput. 15(5) (2011) 66-71.

3. A. Al-Shishtawy and V. Vlassov, Elastman: Autonomic elasticity manager for cloudbased key-value stores, in Proc. 22Nd Int. Symp. High-Performance Parallel and Distributed Computing, HPDC '13 (ACM, New York, NY, USA, 2013), pp. 115-116.

4. W. Wang, B. Li and B. Liang, To reserve or not to reserve: Optimal online multiinstance acquisition in iaas clouds, in Presented as Part of the 10th Int. Conf. Autonomic Computing, Berkeley, CA (2013), pp. 13-22, USENIX.

5. A. Verma, G. Kumar and R. Koller, The cost of reconfiguration in a cloud, in Proc. 11th Int. Middleware Conf. Industrial Track (ACM, New York, NY, USA, 2010), pp. 11-16.

6. Z. Shen, S. Subbiah, X. Gu and J. Wilkes, Cloudscale: Elastic resource scaling for multi-tenant cloud systems, in Proc. 2nd ACM Symp. Cloud Computing, SOCC '11 (ACM, 2011), pp. 5:1-5:14.

7. L. Zhang, X. Meng, S. Meng and J. Tan, K-scope: Online performance tracking for dynamic cloud applications, in Presented as Part of the 10th Int. Conf. Autonomic Computing, Berkeley, CA (2013), pp. 29-32, USENIX.

8. G. Copil, D. Moldovan, H.-L. Truong and S. Dustdar, Multi-level elasticity control of cloud services, in S. Basu, C. Pautasso, L. Zhang and X. Fu (eds.), Service-Oriented Computing, Lecture Notes in Computer Science (Springer, Heidelberg).

9. G. Copil, D. Moldovan, H.-L. Truong and S. Dustdar, Sybl: An extensible language for controlling elasticity in cloud applications, in 13th IEEE/ACM Int. Symp. Cluster, Cloud and Grid Computing (CCGrid), 2013, May 2013, pp. 112-119.

10. D. Trihinas, G. Pallis and M. D. Dikaiakos, JCatascopia: Monitoring elastically adaptive applications in the cloud, in 14th IEEE/ACM Int. Symp. Cluster, Cloud and Grid Computing (2014).

11. D. Moldovan, G. Copil, H.-L. Truong and S. Dustdar, Mela: Monitoring and analyzing elasticity of cloud services, in 2013 IEEE Fifth Int. Conf. Cloud Computing Technology and Science (CloudCom) (2013).

12. G. Copil, D. Trihinas, H.-L. Truong, D. Moldovan, G. Pallis, S. Dustdar and M. Dikaiakos, Advise a framework for evaluating cloud service elasticity behavior, in X. Franch, A. K. Ghose, G. A. Lewis and S. Bhiri (eds.), Service-Oriented Computing, Volume 8831 of Lecture Notes in Computer Science (Springer, Berlin, Heidelberg, 2014), pp. 275-290.

13. OASIS Committee Specification Draft 01. Topology and Orchestration Specification for Cloud Applications Version 1.0. 


\section{G. Copil et al.}

14. OASIS Cloud Application Management Platforms (CAMP), https://www.oasisopen.org/committees/camp.

15. K. V. Mardia, J. T. Kent and J. M. Bibby, Multivariate analysis, Probability and Mathematical Statistics (Academic Press, London, 1979).

16. N. Kaviani, E. Wohlstadter and R. Lea, Profiling-as-a-service: Adaptive scalable resource profiling for the cloud in the cloud, in G. Kappel, Z. Maamar and H. R. Motahari-Nezhad (eds.), Service-Oriented Computing, Vol. 7084 of Lecture Notes in Computer Science (Springer, Berlin, Heidelberg, 2011), pp. 157-171.

17. D. J. Dean, H. Nguyen and X. Gu, Ubl: Unsupervised behavior learning for predicting performance anomalies in virtualized cloud systems, in Proc. 9th Int. Conf. Autonomic Computing, ICAC '12 (ACM, New York, NY, USA, 2012), pp. 191-200.

18. P. Leitner, J. Ferner, W. Hummer and S. Dustdar, Data-driven and automated prediction of service level agreement violations in service compositions, Distributed Parallel Databases 31(3) (2013) 447-470.

19. B. Tang and M. Tang, Bayesian model-based prediction of service level agreement violations for cloud services, in Theoretical Aspects of Software Engineering Conference (TASE), 2014 (2014), pp. 170-176.

20. K. LaCurts, J. C. Mogul, H. Balakrishnan and Y. Turner, Cicada: Introducing predictive guarantees for cloud networks, in 6th USENIX Workshop on Hot Topics in Cloud Computing (HotCloud 14), Philadelphia, June 2014. USENIX.

21. G. Juve and E. Deelman, Automating application deployment in infrastructure clouds, in Proc. 2011 IEEE Third Int. Conf. Cloud Computing Technology and Science, CLOUDCOM '11 IEEE Computer Society, Washington, DC, USA, 2011, pp. 658665.

22. A. Li, X. Zong, S. Kandula, X. Yang and M. Zhang, Cloudprophet: Towards application performance prediction in cloud, in Proc. ACM SIGCOMM 2011 Conf., SIGCOMM '11 (ACM, New York, NY, USA, 2011). 